The Astrophysical Journal, 636:158-171, 2006 January 1

(C) 2006. The American Astronomical Society. All rights reserved. Printed in U.S.A.

\title{
CHEMICAL ENRICHMENT OF THE COMPLEX HOT ISM OF THE ANTENNAE GALAXIES. II. PHYSICAL PROPERTIES OF THE HOT GAS AND SUPERNOVA FEEDBACK
}

\author{
A. Baldi, J. C. Raymond, G. Fabbiano, A. Zezas, and A. H. Rots \\ Harvard-Smithsonian Center for Astrophysics, 60 Garden Street, Cambridge, MA 02138; \\ abaldi@cfa.harvard.edu, jraymond@cfa.harvard.edu, pepi@cfa.harvard.edu, \\ azezas@cfa.harvard.edu, arots@cfa.harvard.edu \\ F. SCHWEIZER \\ Carnegie Observatories, 813 Santa Barbara Street, Pasadena, CA 91101; schweizer@ociw.edu \\ A. R. KING \\ Theoretical Astrophysics Group, University of Leicester, Leicester LE1 7RH, UK; ark@astro.le.ac.uk \\ AND \\ T. J. Ponman \\ School of Physics and Astronomy, University of Birmingham, Birmingham B15 2TT, UK; tjp@star.sr.bham.ac.uk \\ Received 2005 July 26; accepted 2005 August 29
}

\begin{abstract}
We investigate the physical properties of the interstellar medium (ISM) in the merging pair of galaxies known as the Antennae (NGC 4038/4039), using the deep co-added 411 ks Chandra ACIS-S data set. The method of analysis and some of the main results from the spectral analysis, such as metal abundances and their variations from $\sim 0.2$ to $\sim 20-30$ times solar, are described in Paper I (Baldi et al.). In the present paper we investigate in detail the physics of the hot emitting gas, deriving measures for the hot gas mass $\left(\sim 10^{7} M_{\odot}\right)$, cooling times $\left(10^{7}-10^{8} \mathrm{yr}\right)$, and pressure $\left(3.5 \times 10^{-11}-2.8 \times 10^{-10}\right.$ dyne $\left.\mathrm{cm}^{-2}\right)$. In at least one of the two nuclei (NGC 4038), the hot gas pressure is significantly higher than the $\mathrm{CO}$ pressure, implying that shock waves may be driven into the $\mathrm{CO}$ clouds. Comparison of the metal abundances with the average stellar yields predicted by theoretical models of SN explosions points to SNe of Type II as the main contributors of metals to the hot ISM. There is no evidence of any correlation between radio-optical star formation indicators and the measured metal abundances. Although due to uncertainties in the average gas density we cannot exclude that mixing may have played an important role, the short time required to produce the observed metal masses ( $\leqslant 2 \mathrm{Myr}$ ) suggests that the correlations are unlikely to have been destroyed by efficient mixing. More likely, a significant fraction of Type II SN ejecta may be in a cool phase, in grains, or escaping in hot winds. In each case, any such fraction of the ejecta would remain undetectable with soft X-ray observations.
\end{abstract}

Subject headings: galaxies: individual (NGC 4038/4039) — galaxies: interactions — galaxies: peculiar — X-rays: galaxies $-\mathrm{X}$-rays: ISM

\section{INTRODUCTION}

Massive stars deeply influence the baryonic component of the universe. Their ionizing radiation and the supernovae (SNe) return kinetic energy and metal-enriched gas to the interstellar medium (ISM) from which the stars formed (a process usually called "feedback"). Feedback exercises an influence not only on the gas-phase conditions in the immediate environment of the clusters hosting those massive stars (e.g., McKee 1995; Wiseman \& Ho 1998; Pudritz \& Fiege 1999), but also on the phase structure and energetics of the ISM on galactic scales (e.g., McKee \& Ostriker 1977; Norman \& Ferrara 1996) and on the thermodynamics and enrichment of the intergalactic medium (IGM) on scales of several Mpc (e.g., Chiang et al. 1988; Heckman 1999; Aguirre et al. 2001).

The vast range of spatial scales involved is only one of the various difficulties encountered in attempting to study feedback. Even when one restricts the discussion to purely mechanical feedback from $\mathrm{SNe}$ and stellar winds (commonly termed SN feedback), another difficulty is the broad range of complicated gasphase physics, which includes (magneto)hydrodynamic effects such as shocks and turbulence, thermal conduction, and nonionization equilibrium (NEI) emission processes. Yet another complication is that much of the energy and metal-enriched material involved is in the hard-to-observe coronal $\left(T \gtrsim 10^{5} \mathrm{~K}\right)$ and hot $\left(T \gtrsim 10^{6} \mathrm{~K}\right)$ gas phases, requiring the use of space-based far ultraviolet (FUV) and X-ray telescopes.

Several attempts at putting quantitative constraints on the metal enrichment caused by SN feedback in starburst galaxies have been made in the recent past. From a small sample of edge-on starburst galaxies observed by the Röntgensatellit (ROSAT) and the Advanced Satellite for Cosmology and Astrophysics (ASCA), Weaver et al. (2000) obtained abundances completely consistent with solar values and no evidence of supersolar ratios of the $\alpha$-elements with respect to $\mathrm{Fe}$, as required by Type II SN (SN II) feedback (e.g., Gibson et al. 1997). Moreover, they concluded that the technique of measuring abundances through X-ray spectral fitting is highly uncertain because of ambiguities in the fits (e.g., a degeneracy between the temperature and metallicity) and strongly dependent on the model choice (see also Dahlem et al. 1998, 2000; Strickland et al. 2002, 2004). The advent of Chandra (Weisskopf et al. 1996), with its subarcsecond angular resolution, allowed a better subtraction of the point sources from the galactic diffuse emission, simplifying the spectral fitting. However, the low-resolution spectra of the Chandra Advanced CCD Imaging Spectrometer (ACIS) CCDs (Weisskopf et al. 1996) did 


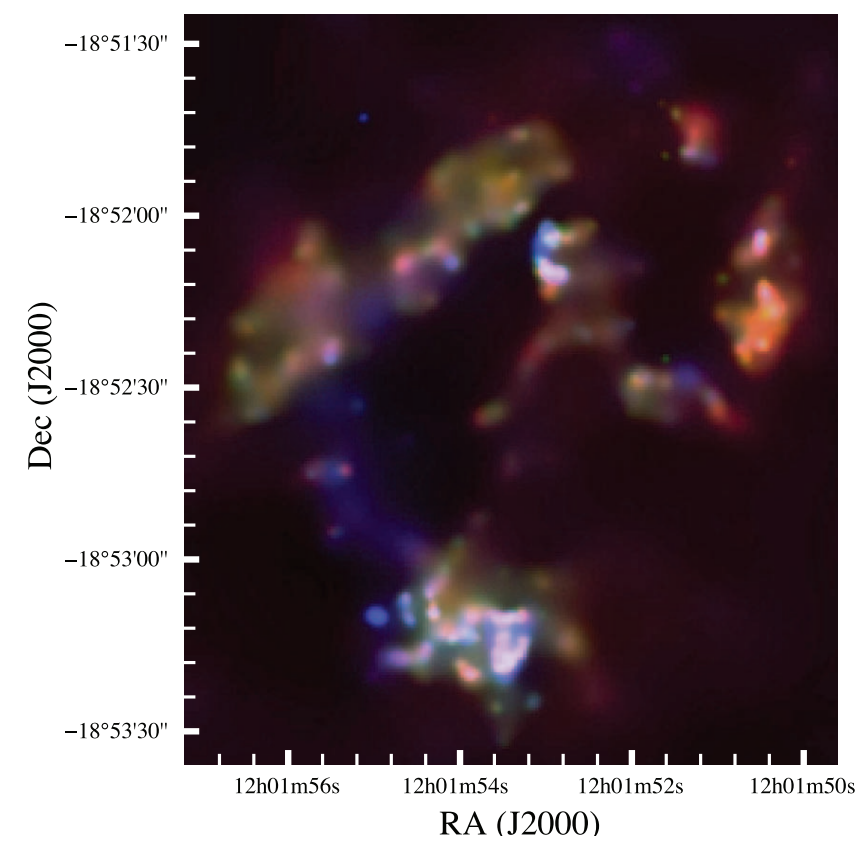

FIG. 1.-Mapped-color broadband smoothed image of the Antennae. The red color channel indicates emission from the $0.3-0.65 \mathrm{keV}$ band, green indicates emission from the $0.65-1.5 \mathrm{keV}$ band, and blue indicates emission from the $1.5-6 \mathrm{keV}$ band.

not allow a dramatic improvement in the accuracy of abundance measures. Notwithstanding the limits of ACIS spectral resolution, Martin et al. (2002), observing the dwarf starburst galaxy NGC 1569 , claimed to obtain ratios of $\alpha$-elements to Fe that were 2-4 times higher than the solar value. They could not put constraints on the individual $\alpha$-element abundances because of degeneracies between metallicity and spectral normalization. Supersolar $\alpha$-element to Fe ratios and supersolar abundances of individual $\alpha$-elements (e.g., $\mathrm{Mg}, \mathrm{Si}, \mathrm{S}$ ) were also reported in other galaxies observed by Chandra; e.g., the face-on spiral galaxies NGC 6946 (Schlegel et al. 2003) and M83 (Soria \& Wu 2003).

While X-ray observations of the halos of nearby edge-on disk galaxies may provide the best single probe of the action of mechanical feedback on galactic scales, observations of the disks of face-on nearby spiral galaxies may provide useful insights into the physics and enrichment of the hot gas. Such observations allow a spatially resolved analysis of the hot ISM in the disk that is much less affected by internal absorption than in the case of edge-on galaxies.

The Antennae are the nearest pair of colliding, relatively faceon spiral galaxies involved in a major merger $(D=19 \mathrm{Mpc}$ for $H_{0}=75 \mathrm{~km} \mathrm{~s}^{-1} \mathrm{Mpc}^{-1}$ ). Hence, this system not only allows a study of the properties of the ISM relatively unaffected by internal absorption, but it also provides a unique opportunity to get the most detailed insight possible into the consequences of a galaxy merger, such as induced star formation and its effect on the ISM.

The presence of an abundant hot ISM in the Antennae was originally suggested by the first Einstein observations of this system (Fabbiano \& Trinchieri 1983) and has since been confirmed by observations with several major X-ray telescopes (ROSAT: Read et al. 1995, Fabbiano et al. 1997; ASCA: Sansom et al. 1996). The first Chandra ACIS observation of the Antennae in 1999 gave us for the first time a detailed look at this hot ISM, revealing a complex, diffuse, and soft emission component responsible

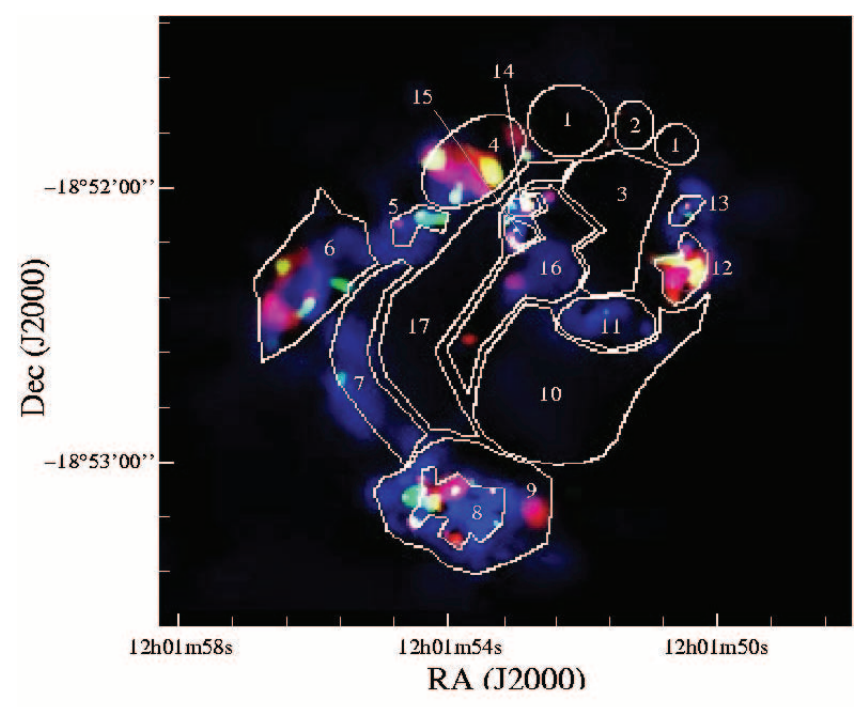

FIG. 2.-Mapped-color line-strength image of the Antennae. Red represents emission from the $\mathrm{Fe} \mathrm{K}$ line blend (plus $\mathrm{O}$ and $\mathrm{Ne}$ ), green represents that from the $\mathrm{Mg}$ line, and blue represents that from the Si line (for details, see text). The 17 regions used for a preliminary subdivision of the hot ISM (performed in Paper I) are marked in white.

for about half of the detected X-ray photons from the two merging galaxies (Fabbiano et al. 2001; Fabbiano et al. 2003, hereafter F03). The spatial resolution of Chandra is at least 10 times superior to that of any previous X-ray observatory, which allows us to resolve the emission on physical scales of $\sim 75 \mathrm{pc}$ (for $D=19 \mathrm{Mpc}$ ) and to detect and subtract individual pointlike sources (most likely X-ray binaries; see Zezas et al. 2002). At the same time, the spectral resolution of ACIS also allows us to study for the first time the X-ray spectral properties of the various emission regions, providing important additional constraints on their nature.

While the first Chandra data set demonstrated the richness of the ISM in the Antennae, the number of detected photons was insufficient to study its detailed small-scale morphology and spectral properties. A deep monitoring observing campaign of the Antennae with the Chandra ACIS has produced a detailed and rich data set. A first look at this data set revealed a complex diffuse emission, with signatures of strong line emission coming from some of the hot ISM regions, pointing to high $\alpha$-element abundances (Fabbiano et al. 2004). This paper focuses on the physical properties of the hot gas and its enrichment by SN explosions. In a companion paper we describe the data analysis procedure, the creation of broadband mapped-color images of the diffuse emission, and the performed spectral analysis (Baldi et al. 2006, hereafter Paper I).

This paper is organized as follows. In $\S 2$ we briefly review the main results of Paper I. In $\S 3$ we derive the hot gas parameters, such as pressure and density, from the spectral fits. In $\S 4$ we discuss the main results derived from the abundance measures, focusing especially on the supernova feedback. In $\S 5$ we present some further insights into the physical properties of the hot ISM, and in $\S 6$ we summarize the main results.

\section{MAPPED-COLOR IMAGES OF THE DIFFUSE EMISSION AND MAIN RESULTS OF THE SPECTRAL ANALYSIS}

NGC 4038/4039 was observed with Chandra ACIS-S seven times during the period between 1999 December and 2002 November, for a total of $\sim 411 \mathrm{ks}$. The data products were analyzed 


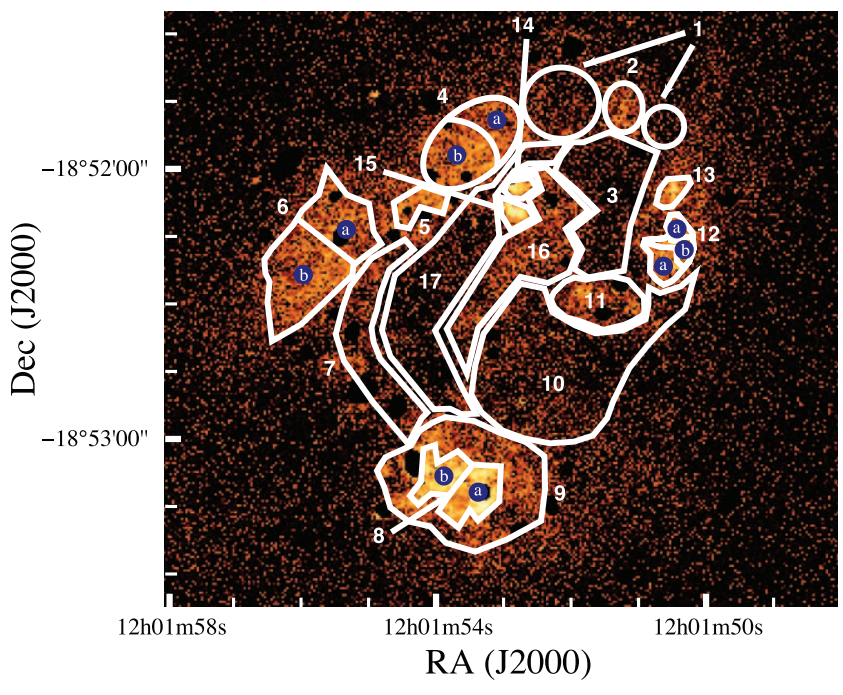

FIG. 3.- Image of the Antennae in the $0.3-6 \mathrm{keV}$ band, obtained after removing the $\geq 3 \sigma$ point sources. The 21 regions used for the spectral analysis of the hot ISM are marked in white.

with the Chandra X-Ray Center (CXC) CIAO version 3.0.1 software and the XSPEC package. Full details about the data processing and reduction are given in Paper I.

Paper I presents a mapped-color image of the diffuse emission in the Antennae, representing contributions from three different energy bands: $0.3-0.65,0.65-1.5$, and $1.5-6 \mathrm{keV}$. Before combining the images from the three bands, all point sources were subtracted and an adaptive Gaussian kernel was applied to each of the three images. Figure 1 reproduces the mapped-color image for the reader's convenience. Red denotes soft X-ray emission $(0.3-0.65 \mathrm{keV})$, green represents intermediate-energy emission $(0.65-1.5 \mathrm{keV})$, and blue shows hard X-ray emission (1.5-6 keV).
A similar procedure was applied to generate a line-strength map for the emission lines from $\mathrm{O}+\mathrm{Fe}+\mathrm{Ne}, \mathrm{Mg}$, and $\mathrm{Si}$ in different regions of the hot ISM. Figure 2 shows this second map, where red, green, and blue represent $\mathrm{O}+\mathrm{Fe}+\mathrm{Ne}$ emission $(0.6-1.16 \mathrm{keV})$, $\mathrm{Mg}$ emission (1.27-1.38 keV), and Si emission (1.75-1.95 keV), respectively.

Both the mapped-color image of the diffuse emission and the line-strength map guided us in selecting 21 spectrally similar regions for a proper spectral analysis. These regions are shown in Figure 3. The method used for the spectral extraction and analysis is fully described in Paper I. Table 1 reviews the main results of the spectral analysis conducted so far. In this table, the goodness of fit ( $\chi_{\text {red }}^{2}$ over the number of degrees of freedom [dof]), absorbing column density $N_{\mathrm{H}}$, temperature(s) $k T$, abundances of neon, magnesium, silicon, and iron, and power-law photon index $\Gamma$ (if a power-law component is necessary) are summarized together with the "best-fit model" (as defined in $\S 5.2$ of Paper I) for each region.

Almost all regions could be fitted with a single thermal component. Only region $4 \mathrm{~b}$ required two thermal components with different values of $k T$; in this region, we could not constrain the low $k T$ value (although we found a minimum in the $\chi^{2}$ statistics at $k T=0.20 \mathrm{keV}$ ), and we could put only a lower limit $(k T>0.54 \mathrm{keV})$ to the higher temperature component. Almost all the single-temperature regions required a temperature of $k T \sim 0.6 \mathrm{keV}$ to fit the data, except for regions $5,8 \mathrm{~b}$, and 14 $(k T \sim 0.3 \mathrm{keV})$. Considering all the regions analyzed, the temperatures (both in the single- and two-temperature cases) range from $\sim 0.2$ to $\sim 0.8 \mathrm{keV}$ (including the errors). Our analysis yielded metal abundances that have acceptable constraints in the majority of regions and led to the most important, and somewhat unexpected, result of our spectral analysis: the abundances of silicon and magnesium cover a wide range of values, ranging from the very low values found in regions $2,6 \mathrm{a}, 12 \mathrm{a}$, and $12 \mathrm{~b}$ $\left(Z \sim 0.2 Z_{\odot}\right)$ to the very high values observed in region 5

TABLE 1

Best-Fit Parameters for the Diffuse Emission of the Antennae

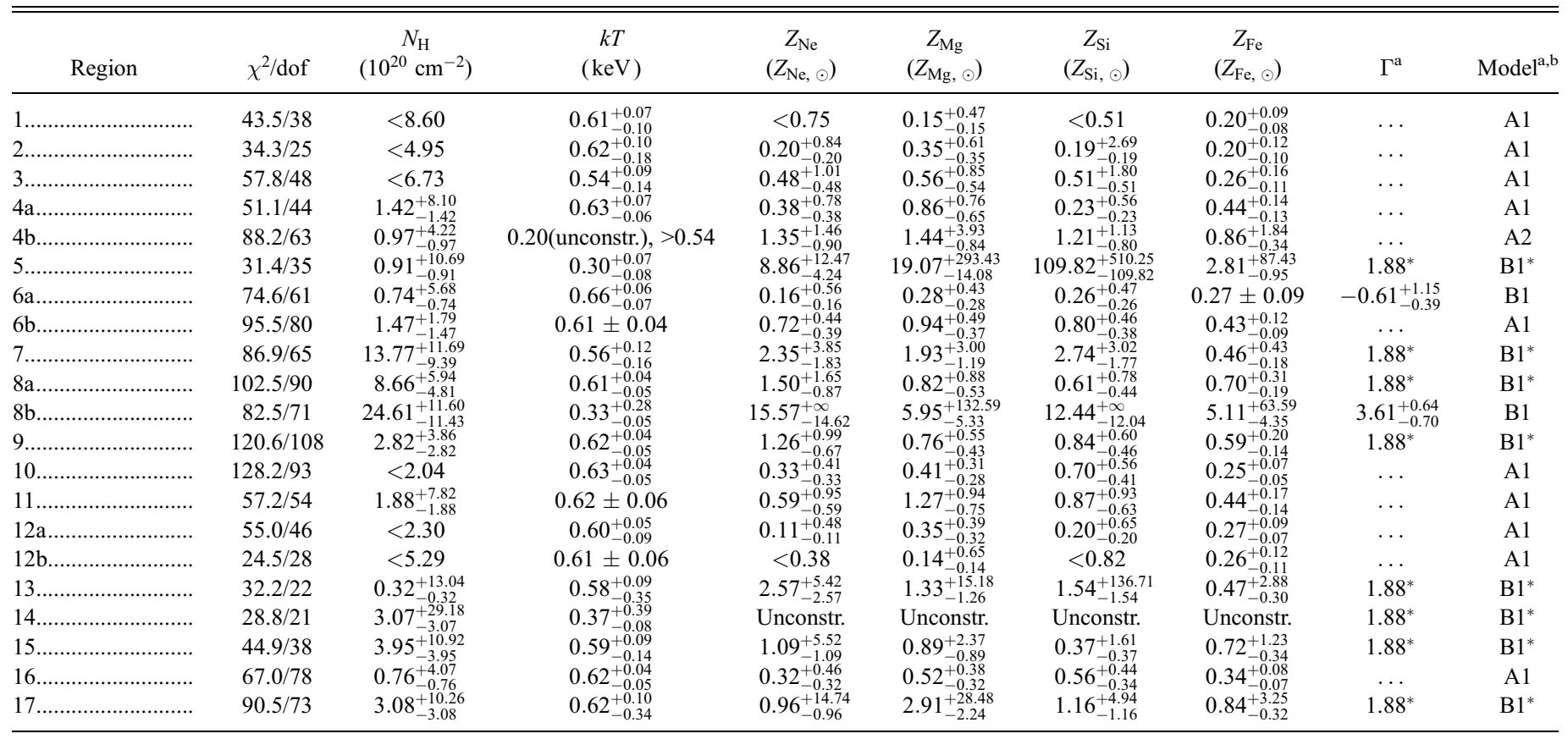

Note.-Errors are $1 \sigma$ for all interesting parameters; see text for details.

a Asterisk $\left({ }^{*}\right)$ indicates frozen photon index.

b XSPEC models: A1 = $\operatorname{wabs}(\operatorname{wabs}(\operatorname{vapec})) ; \mathrm{A} 2=\operatorname{wabs}(\operatorname{wabs}(\operatorname{vapec}+\operatorname{vapec})) ; \mathrm{B} 1=\operatorname{wabs}($ wabs $(\operatorname{vapec}+\operatorname{powerlaw})) ; \mathrm{B} 2=\operatorname{wabs}($ wabs $(\operatorname{vapec}+\operatorname{vapec}+$ powerlaw)). 
TABLE 2

Emission Parameters for the Diffuse Emission of the Antennae

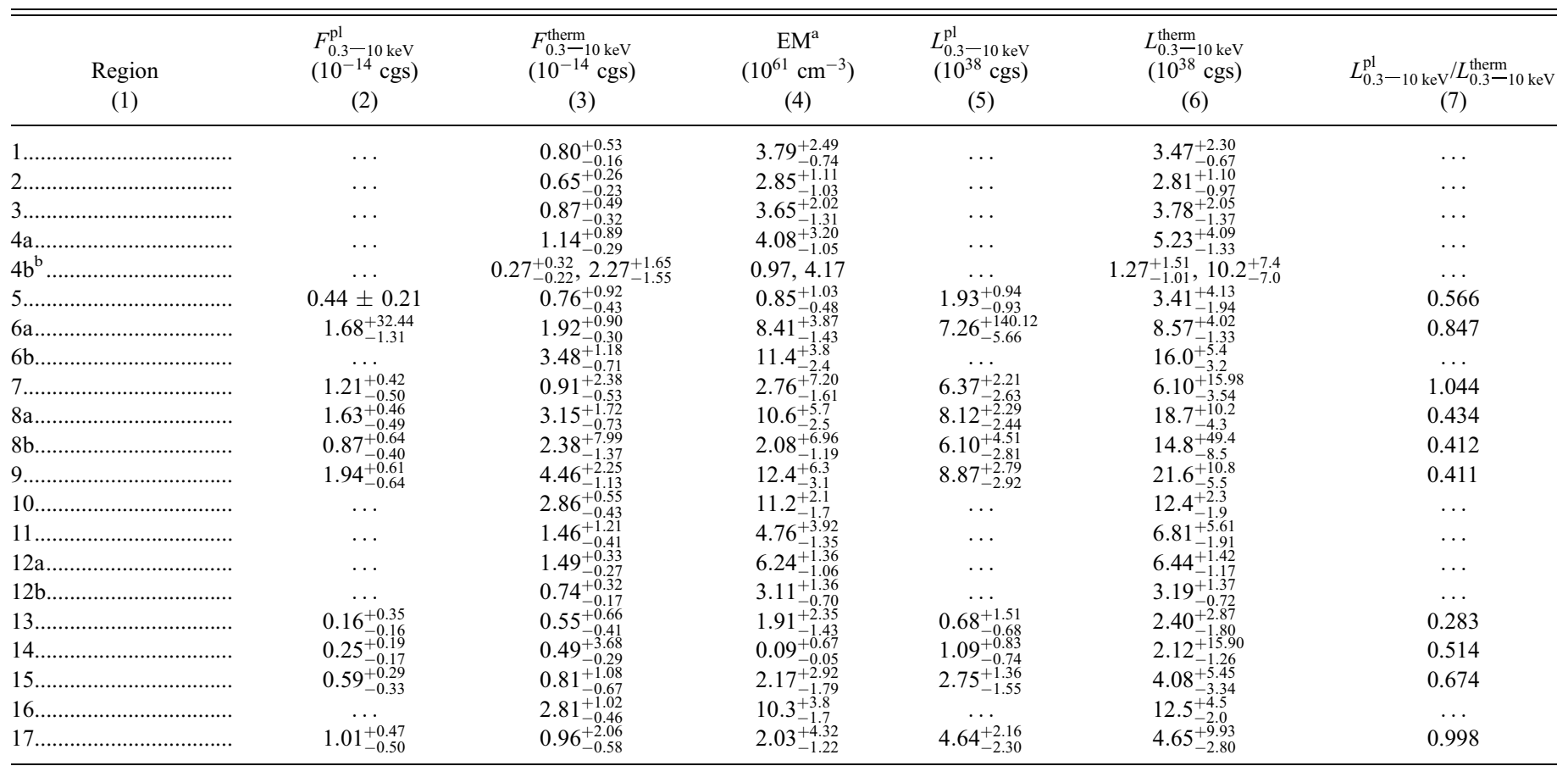

${ }^{\text {a }}$ Emission measure defined as $\mathrm{EM}=n^{2} V$.

${ }^{\mathrm{b}}$ In the case of a two-temperature gas, the quantities are listed separately for both components: lower and higher temperature, respectively. For the calculation of EM we assumed the best-fit value of $k T_{1}$ and $k T_{2}$; it was not possible to determine a confidence range for EM because of the indetermination in both temperatures.

( $Z \gtrsim 20 Z_{\odot}$ ). The intrinsic absorption throughout the hot ISM is generally low, with $N_{\mathrm{H}} \sim 10^{20} \mathrm{~cm}^{-2}$ typical and often consistent with zero. The exceptions are the southern nucleus (regions $8 \mathrm{a}$ and $8 \mathrm{~b}$ ) and region 7 , which is significantly obscured also in the optical. In the optical and near-infrared, region 7 corresponds to the "overlap region," where the most active star formation is now occurring (Mirabel et al. 1998; Wilson et al. 2000; Zhang et al. 2001). These three regions show intrinsic absorption of $\sim 1-2 \times 10^{21} \mathrm{~cm}^{-2}$.

\section{HOT GAS PARAMETERS}

All the calculations performed in this section use the parameters obtained from the best-fit spectral models and listed in Table 1. Table 2 gives the detailed emission parameters derived from the spectral analysis of each region. The observed flux in the $0.3-10 \mathrm{keV}$ band is listed for both the power law (if present) and the thermal emission in columns (2) and (3), respectively. The emission measure $\left(\mathrm{EM}=n^{2} V\right)$ is listed in column (4). For each region we present also the intrinsic (absorptioncorrected for $N_{\mathrm{H}}$ ) luminosity in the $0.3-10 \mathrm{keV}$ band for both the power law (if present) and the thermal emission (cols. [5] and [6]). Column (7) gives the ratio between the intrinsic luminosities in the $0.3-10 \mathrm{keV}$ band of the power-law and thermal components. In the case of region $4 \mathrm{~b}$, which was modeled with two temperatures, we were unable to determine a confidence range for EM because of the lack of constraints on both temperatures $\left(k T_{1}\right.$ unconstrained and $k T_{2}>0.54 \mathrm{keV}$ at $1 \sigma$ ). Therefore, we give only the values corresponding to the best-fit $k T_{1}$ and $k T_{2}$ (corresponding to 0.20 and $0.60 \mathrm{keV}$, respectively).

From these parameters and those listed in Table 1, we can estimate various properties of the emitting plasma, such as electron density $n_{e}\left(\approx n_{\mathrm{H}}\right)$, thermal energy content $E_{\mathrm{th}}$, cooling time $\tau_{c}$, and pressure $p$. These quantities are given in Table 3 . The calcu- lation of these parameters depends on the emitting volume and on the filling factor $\eta$ of the hot gas. Since we observe regions projected on the plane of the sky, we can only measure the "footprint" of each emitting region. To estimate the emitting volumes we adopted the following procedure. For all singletemperature regions, we assumed a depth of $200 \mathrm{~K}$ pc for the emitting volume, where $K$ is a variable parameter (the numbers given in Table 3 assume $K=1$, corresponding to the typical depth of a spiral disk). In the case of region $4 b$, where we have two temperatures, we assumed that the high- and low-temperature components are in pressure equilibrium and derived the thicknesses for the volumes occupied by these two phases from the best-fit values of $k T$, imposing the condition that they add up to a total value of $200 \mathrm{~K}$ pc. The physical area covered by each region scaled to the distance of the Antennae is given in column (2). Column (3) lists the thermal gas electron density $n_{e}$. This quantity depends both on the depth-variable parameter $K$ and on the filling factor $\eta$ of the gas and is proportional to $\eta^{-1 / 2} K^{-1 / 2}$. The thermal energy $E_{\text {th }}$ and the cooling time $\tau_{c}$ (cols. [4] and [5], respectively) were estimated following Tucker (1975). Both these quantities are proportional to $\eta^{1 / 2} K^{1 / 2}$. The thermal pressure of the hot gas, expressed in dyne $\mathrm{cm}^{-2}$, is listed in column (6). The pressure ( $p=2 n k T)$, which is directly proportional to the density $n_{e}$, has the same dependence on the depth-variable parameter $K$ and filling factor $\eta$ as the density $\left(\eta^{-1 / 2} K^{-1 / 2}\right)$. The filling factor $\eta$ is assumed to be unity in our calculations. A filling factor $\eta \lesssim 1$ for the hot phase of the ISM is plausible from recent results coming from three-dimensional hydrodynamical simulations ( $\eta$ ranging between 0.17 and 0.44 for SN rate between Galactic and 16 times the Galactic value; see, e.g., de Avillez \& Breitschwerdt 2004 and references therein). However, our parameters have only a slight dependence on it $\left(\propto \eta^{-1 / 2}\right.$ or $\left.\propto \eta^{1 / 2}\right)$; hence, our estimates are precise within a factor of 2 or less. As described above for the emission measure EM, in region $4 \mathrm{~b}$ we 
TABLE 3

Hot Gas Parameters

\begin{tabular}{|c|c|c|c|c|c|}
\hline $\begin{array}{l}\text { Region } \\
\text { (1) }\end{array}$ & $\begin{array}{c}\text { Area } \\
\left(\mathrm{kpc}^{2}\right) \\
(2)\end{array}$ & $\begin{array}{c}n_{e} \\
\left(10^{-2} \mathrm{~cm}^{-3}\right) \\
(3)\end{array}$ & $\begin{array}{c}E_{\mathrm{th}} \\
\left(10^{53} \mathrm{ergs}\right) \\
(4)\end{array}$ & $\begin{array}{c}\tau_{c} \\
\left(10^{7} \mathrm{yr}\right) \\
(5)\end{array}$ & $\begin{array}{c}p \\
\left(10^{-11} \text { dyne } \mathrm{cm}^{-2}\right) \\
(6)\end{array}$ \\
\hline ......... & 2.260 & $5.34_{-1.54}^{+1.54}$ & $20.8_{-5}^{+9.0}$ & $19.0^{+14.7}$ & $10.4^{+4.6}$ \\
\hline 2.2. & 0.597 & $9.01_{-1.81}^{+1.65}$ & $9.41_{-4.08}^{+3.26}$ & $10.6_{-6.3}^{+10.4}$ & $17.9_{-7.8}^{+6.6}$ \\
\hline З & 3.361 & $4.30_{-0.86}^{+1.81}$ & $22.0_{-90}^{+10.08}$ & $18.4_{-11.3}^{-6.3}$ & $7.43_{-3.03}^{+3.38}$ \\
\hline 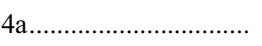 & 0.960 & $8.50_{-1.17}^{+2.86}$ & $14.5_{-3.2}^{+9.0}$ & $8.79_{-4.95}^{+8.68}$ & $17.1_{-3.7}^{+8.03}$ \\
\hline $4 b^{\mathrm{a}}$ & 1.453 & $21.2,7.08$ & $0.44,17.0$ & $1.10,5.28$ & 13.6 \\
\hline 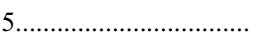 & 0.483 & $5.48_{-1.88}^{+2.67}$ & $2.24_{-1.16}^{+1.86}$ & $2.08_{-1.63}^{+6.76}$ & $5.26_{-2.73}^{+4.39}$ \\
\hline 6а............................... & 1.405 & $10.1_{-0.9}^{+2.10}$ & $26.4_{-4.9}^{+8.46}$ & $9.76_{-4.35}^{+5.63}$ & $21.3_{-3.9}^{+6.8}$ \\
\hline 6b................................ & 2.551 & $8.71_{-0.95}^{+1.36}$ & $38.2_{-6.4}^{+8.9}$ & $7.57_{-2.86}^{+4.09}$ & $17.0_{-2.9}^{+3.9}$ \\
\hline 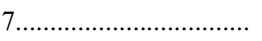 & 2.351 & $4.47_{-1.58}^{+4.02}$ & $16.6_{-8.9}^{+21.7}$ & $8.62_{-7.51}^{+38.80}$ & $8.01_{-4.31}^{+10.46}$ \\
\hline 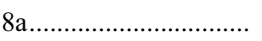 & 0.856 & $14.5_{-1.8}^{+3.58}$ & $21.4_{-4.2}^{+6.9}$ & $3.63_{-1.74}^{+2.51}$ & $28.3_{-5.5}^{+9.31}$ \\
\hline $8 \mathrm{~b}$ & 0.756 & $6.84_{-2.37}^{+7.43}$ & $4.81_{-2.67}^{+13.75}$ & $1.03_{-0.92}^{+8.31}$ & $7.22_{-3.21}^{+20.64}$ \\
\hline 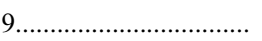 & 4.528 & $6.84_{-0.93}^{+1.55}$ & $54.2_{-11.2}^{+16.5}$ & $7.95_{-3.74}^{+5.97}$ & $13.6_{-2.8}^{+4.1}$ \\
\hline $10 \ldots \ldots \ldots \ldots \ldots \ldots \ldots . .$. & 7.783 & $4.94_{-0.39}^{+0.45}$ & $68.3_{-10.4}^{+11.0}$ & $17.5_{-5.0}^{+6.4}$ & $9.96_{-1.52}^{+1.60}$ \\
\hline $11 \ldots \ldots \ldots \ldots$ & 1.317 & $7.84_{-1.20}^{+2.75}$ & $18.0_{-4.2}^{+8.7}$ & $8.38_{-4.86}^{+8.89}$ & $15.6_{-3.7}^{+7.4}$ \\
\hline 12a & 0.583 & $13.5_{-1.2}^{+1.4}$ & $13.3_{-3.0}^{+2.6}$ & $6.54_{-2.39}^{+3.02}$ & $25.9_{-5.8}^{+5.1}$ \\
\hline $12 \mathrm{~b}$ & 0.266 & $14.1_{-1.7}^{+2.8}$ & $6.46_{-1.34}^{+2.04}$ & $6.42_{-2.86}^{+4.49}$ & $27.5_{-5.7}^{+8.9}$ \\
\hline $13 \ldots \ldots \ldots \ldots$ & 0.269 & $11.0_{-5.5}^{+5.4}$ & $4.85_{-3.89}^{+3.50}$ & $6.40_{-5.82}^{+37.70}$ & $20.4_{-16.4}^{+14.8}$ \\
\hline 14 & 0.171 & $2.98_{-1.08}^{+5.70}$ & $1.43_{-1.16}^{+1.74}$ & $2.14_{-2.09}^{+9.54}$ & $3.53_{-1.77}^{+17.58}$ \\
\hline 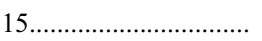 & 0.261 & $\begin{array}{l}11.9_{-6.9}^{+6.30} \\
\end{array}$ & $3.25_{-1.59}^{+5.87}$ & $2.52_{-1.97}^{+36.50}$ & $22.5_{-15.3}^{+17.1}$ \\
\hline 16 & 3.391 & $7.20_{-0.62}^{+1.91}$ & $42.7_{-6.8}^{+10.4}$ & $10.8_{-4.1}^{+5.2}$ & $14.3_{-2.3}^{+3.3}$ \\
\hline 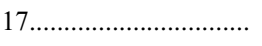 & 5.363 & $2.54_{-0.94}^{+1.95}$ & $23.8_{-17.0}^{+25.1}$ & $16.2_{-14.7}^{+67.6}$ & $5.04_{-3.61}^{+5.30}$ \\
\hline
\end{tabular}

${ }^{\mathrm{a}}$ In the case of a two-temperature gas, we calculated $n_{e}, E_{\mathrm{th}}$, and $\tau_{c}$ for both components (assuming the best-fit temperatures and pressure equilibrium), given for the lower and higher temperatures, respectively. It was not possible to determine a confidence range for those parameters and for $p$ because of the indetermination in both temperatures.

were unable to compute a confidence range for $n_{e}, E_{\mathrm{th}}, \tau_{c}$, and $p$ because of the indeterminacy of the two temperatures. The values of these parameters for region $4 \mathrm{~b}$ correspond to the bestfit values of $k T_{1}$ and $k T_{2}$. Metz et al. (2004) found somewhat larger thermal energies for regions similar to ours. This probably results from their smaller density estimates.

The calculated cooling times, in the $10^{7}-10^{8} \mathrm{yr}$ range, are in agreement with the results of F03. The pressure is generally on the order of a few times $10^{-11}$ dyne $\mathrm{cm}^{-2}$ but reaches higher values (a few times $10^{-10}$ dyne $\mathrm{cm}^{-2}$ ) in the two nuclei and also in the regions corresponding to the hot spot regions R1 and R2 of F03. These new values are in excess of those found in F03, possibly reflecting the fact that larger areas were averaged in that work. To test this hypothesis we extracted a spectrum for each of these regions using the same region files as in F03. The pressures we obtain for all four regions are, indeed, lower than our new

TABLE 4

Hot Gas Masses for Hydrogen and Heavy Elements

\begin{tabular}{|c|c|c|c|c|c|}
\hline Region & $\begin{array}{c}\text { Total Mass } \\
\left(M_{\odot}\right)\end{array}$ & $\begin{array}{c}\text { Ne Mass } \\
\left(M_{\odot}\right)\end{array}$ & $\begin{array}{c}\text { Mg Mass } \\
\left(M_{\odot}\right)\end{array}$ & $\begin{array}{l}\text { Si Mass } \\
\left(M_{\odot}\right)\end{array}$ & $\begin{array}{c}\text { Fe Mass } \\
\left(M_{\odot}\right)\end{array}$ \\
\hline 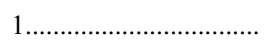 & $5.9 \times 10^{5}$ & $<1.1 \times 10^{3}$ & $8.2 \times 10^{1}$ & $<3.0 \times 10^{2}$ & $3.1 \times 10^{2}$ \\
\hline 2 & $2.6 \times 10^{5}$ & $1.3 \times 10^{2}$ & $8.5 \times 10^{1}$ & $5.0 \times 10^{1}$ & $1.4 \times 10^{2}$ \\
\hline 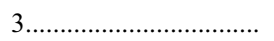 & $7.1 \times 10^{5}$ & $8.5 \times 10^{2}$ & $3.7 \times 10^{2}$ & $3.6 \times 10^{2}$ & $4.8 \times 10^{2}$ \\
\hline 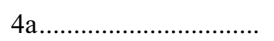 & $4.0 \times 10^{5}$ & $3.8 \times 10^{2}$ & $3.2 \times 10^{2}$ & $9.2 \times 10^{1}$ & $4.6 \times 10^{2}$ \\
\hline $4 \mathrm{~b}$ & $5.3 \times 10^{5}$ & $1.8 \times 10^{3}$ & $7.1 \times 10^{2}$ & $6.4 \times 10^{2}$ & $1.2 \times 10^{3}$ \\
\hline 5 & $1.3 \times 10^{5}$ & $2.9 \times 10^{3}$ & $2.3 \times 10^{3}$ & $1.4 \times 10^{4}$ & $9.5 \times 10^{2}$ \\
\hline 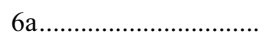 & $7.0 \times 10^{5}$ & $2.8 \times 10^{2}$ & $1.8 \times 10^{2}$ & $1.8 \times 10^{2}$ & $4.9 \times 10^{2}$ \\
\hline 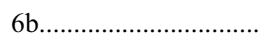 & $1.1 \times 10^{6}$ & $2.0 \times 10^{3}$ & $9.5 \times 10^{2}$ & $8.7 \times 10^{2}$ & $1.2 \times 10^{3}$ \\
\hline 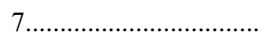 & $5.2 \times 10^{5}$ & $3.0 \times 10^{3}$ & $9.2 \times 10^{2}$ & $1.4 \times 10^{3}$ & $6.2 \times 10^{2}$ \\
\hline 8a & $6.1 \times 10^{5}$ & $2.3 \times 10^{3}$ & $4.6 \times 10^{2}$ & $3.7 \times 10^{2}$ & $1.1 \times 10^{3}$ \\
\hline $8 \mathrm{~b}$ & $2.5 \times 10^{5}$ & $9.8 \times 10^{3}$ & $1.4 \times 10^{3}$ & $3.2 \times 10^{3}$ & $3.4 \times 10^{3}$ \\
\hline 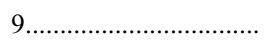 & $1.5 \times 10^{6}$ & $4.8 \times 10^{3}$ & $1.1 \times 10^{3}$ & $1.3 \times 10^{3}$ & $2.3 \times 10^{3}$ \\
\hline $10 \ldots$ & $1.9 \times 10^{6}$ & $1.6 \times 10^{3}$ & $7.2 \times 10^{2}$ & $1.3 \times 10^{3}$ & $1.2 \times 10^{3}$ \\
\hline $11 \ldots$ & $5.1 \times 10^{5}$ & $7.4 \times 10^{2}$ & $6.0 \times 10^{2}$ & $4.4 \times 10^{2}$ & $5.8 \times 10^{2}$ \\
\hline 12a & $3.9 \times 10^{5}$ & $1.1 \times 10^{2}$ & $1.3 \times 10^{2}$ & $7.7 \times 10^{1}$ & $2.7 \times 10^{2}$ \\
\hline 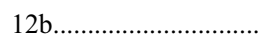 & $1.8 \times 10^{5}$ & $<1.7 \times 10^{2}$ & $2.4 \times 10^{1}$ & $<1.5 \times 10^{2}$ & $1.3 \times 10^{2}$ \\
\hline 13 & $1.5 \times 10^{5}$ & $9.3 \times 10^{2}$ & $1.8 \times 10^{2}$ & $2.2 \times 10^{2}$ & $1.8 \times 10^{2}$ \\
\hline 14 & $2.5 \times 10^{4}$ & $\ldots$ & $\ldots$ & & 1.0 \\
\hline 15 & $1.5 \times 10^{5}$ & $4.1 \times 10^{2}$ & $1.3 \times 10^{2}$ & $5.6 \times 10^{1}$ & $2.9 \times 10^{2}$ \\
\hline 16 & $1.2 \times 10^{6}$ & $9.5 \times 10^{2}$ & $5.8 \times 10^{2}$ & $6.7 \times 10^{2}$ & $1.1 \times 10^{3}$ \\
\hline $17 \ldots \ldots$ & $6.7 \times 10^{5}$ & $1.6 \times 10^{3}$ & $1.8 \times 10^{3}$ & $7.7 \times 10^{2}$ & $1.5 \times 10^{3}$ \\
\hline
\end{tabular}


estimates in the corresponding regions, although still higher than the pressure estimates in F03. F03 argued for a possible pressure equilibrium between the hot gas and the cold molecular clouds. Zhu et al. (2003) derived from CO measurements a pressure of $4.2 \times 10^{-11}$ dyne $\mathrm{cm}^{-2}$ for the northern nucleus (NGC 4038) and of $3.1 \times 10^{-11}$ dyne $\mathrm{cm}^{-2}$ for the southern nucleus (NGC 4039). Our observations suggest that the hot gas pressure is a few times higher than the $\mathrm{CO}$ estimate for the northern nucleus and almost an order of magnitude higher in the southern nucleus. Such a large pressure difference would imply that shock waves are being driven into the $\mathrm{CO}$ clouds. The hot ISM may therefore in part be responsible for compressing and fragmenting the $\mathrm{CO}$ clouds, triggering star formation. However, we must bear in mind the large uncertainties in the estimated pressures, due to the assumptions we had to make on the emitting volume and the smallscale properties of the clouds.

Table 4 presents our estimates of the total mass of hot gas and of the masses of the individual elements $\mathrm{Ne}, \mathrm{Mg}, \mathrm{Si}$, and $\mathrm{Fe}$ for each region. The calculation of these individual-element masses was performed using the Anders \& Grevesse (1989) compilation of meteoritic abundances. All values in Table 4 are dependent on both $K$ and $\eta$ and are proportional to $\eta^{1 / 2} K^{1 / 2}$. For both the northern and the southern nuclei, our total mass estimates are a factor of 2 smaller than the values previously obtained in F03. This discrepancy may be due to differences in the best-fit models and to the sizes of the regions used for the spectrum extraction, which were larger in the F03 analysis. The total mass of hot gas derived by adding the contributions from all individual regions is $\sim 1.3 \times 10^{7} M_{\odot}$. For the individual elements we obtain total masses of $\sim 3.4 \times 10^{4} M_{\odot}$ for $\mathrm{Ne}, \sim 1.3 \times 10^{4} M_{\odot}$ for $\mathrm{Mg}, \sim 2.6 \times 10^{4} M_{\odot}$ for $\mathrm{Si}$, and $\sim 1.8 \times 10^{4} M_{\odot}$ for $\mathrm{Fe}$.

It is worthwhile to note that, if we adopted the smaller distance of $\sim 14 \mathrm{Mpc}$ for the Antennae, as derived from the tip of the red giant branch by Saviane et al. (2004), some of the above physical parameters would change. In particular, the thermal energies, luminosities, and masses would be smaller by a factor of almost 2 , while all other listed quantities would remain unvaried.

\section{ABUNDANCES}

The unprecedented high angular resolution and sensitivity of Chandra, in conjunction with the long integrated exposure time of our observations, allows us to analyze in detail the properties of the hot ISM in the Antennae, down to regions measuring a few hundred pc in size. On the basis of only the first 75 ks observation of 1999 December, F03 were unable to constrain the variety of metal abundances in these regions. Indeed, the measure of hot gas elemental abundances in star-forming galaxies has always been a challenging task (e.g., Strickland \& Stevens 1998, 2000; Dahlem et al. 1998, 2000; Weaver et al. 2000; Strickland et al. 2002, 2004). However, the new, deeper total exposure has allowed us, within the limitations of the low spectral resolution of the ACIS CCDs, to place better constraints on the abundances than in the past. The method we have used to determine the abundances and their relative confidence ranges, described in Paper I, is conservative enough to take into account both statistical and systematic biases that may affect the abundance measures in our low-resolution spectra, especially regarding the biases deriving from model choice and from the metallicity-temperature degeneracy. In the past, spatially resolved measurements of individual elemental abundances were performed on megaparsec-size regions in the hot intracluster medium of galaxy clusters (e.g., Mushotzky et al. 1996; Finoguenov et al. 2000) and, on a much smaller scale, in elliptical galaxies (e.g., Finoguenov et al. 2002). With the new Chandra data for the Antennae, we are now trying to explore the "local" chemical enrichment of the hot ISM by supernovae within two interacting galaxies.

As discussed in Paper I, the elemental abundances of $\mathrm{Ne}$ IX, $\mathrm{Mg}$ XI, Si XIII, and Fe L that we measure in the hot ISM of the Antennae are generally consistent with the stellar abundances measured from optical data $(\sim 0.5$ solar; Fritze-v. Alvensleben 1998), except for a few regions (4b, 5, 7, 8a, and 8b) where the measured abundances are clearly in excess of this value and, in the case of region 5, even significantly supersolar. Indeed, difficulties in measuring the abundances through X-ray spectral fitting of low-resolution spectra are well documented in the literature (e.g., Weaver et al. 2000). Our very conservative approach, however, did not prevent us from locating peculiar regions with bona fide supersolar abundances. While all the other regions have metallicities consistent with solar (within the errors), region 5 shows particularly enhanced abundances for three out of the four elements we measured $\left(Z_{\mathrm{Ne}}>3.9, Z_{\mathrm{Mg}}>\right.$ 3.8 , and $Z_{\mathrm{Fe}}>1.3$, at $90 \%$ confidence level ). Such high X-rayderived metallicities of $\mathrm{Fe}$ and especially of the $\alpha$-elements are quite rare and have not often been found in previous observations of other galaxies. This could be due both to the peculiarity of region 5 and to the fact that constraining abundances through $\mathrm{X}$-ray fitting of low-resolution spectra is always a challenging task. From a Chandra observation of the face-on spiral NGC 6946, Schlegel et al. (2003) found an abundance of $\mathrm{Si}=$ $3.00_{-1.90}^{+1.95}$ for a single-temperature model fit of the diffuse emission in this galaxy. Although the errors they quote are at $90 \%$, they estimated this confidence level considering only two interesting parameters, a method a lot less conservative than ours. Hints of supersolar values of the $\alpha$-element abundances were also found in the starburst spiral galaxy M83 by Soria \& Wu (2003). However, these authors did not fit the abundances, but simply realized that a single-temperature thermal model with the $\alpha$-element abundances fixed at supersolar values and an $\alpha$-element-to-Fe ratio higher than solar (e.g., $Z_{\mathrm{Ne}}=2.0, Z_{\mathrm{Mg}}=$ $\left.2.5, Z_{\mathrm{Si}}=3.0, Z_{\mathrm{Fe}}=0.9\right)$ clearly gives a better fit $\left(\chi_{\text {red }}^{2} \sim 0.99\right)$ than a model with all the elements fixed at $\operatorname{solar}\left(\chi_{\text {red }}^{2} \sim 1.42\right)$. All the other measurements derived from Chandra ACIS observations of starburst galaxies or ultraluminous infrared galaxies (ULIRGs) and presented recently in the literature led to abundances consistent with solar (e.g., the ULIRG sample of Huo et al. 2004; the edge-on starburst sample of Strickland et al. 2004) or clearly subsolar (e.g., NGC 253; Strickland et al. 2002) values.

The ratios between elemental abundances in each region can be related to the type of supernovae generating the metals. If the elemental enrichment results from Type II supernovae, one would expect $[\mathrm{Ne} / \mathrm{Fe}]$ and $[\mathrm{Mg} / \mathrm{Fe}]$ values approaching 0.3 and $[\mathrm{Si} / \mathrm{Fe}]$ values approaching 0.5 on average; the corresponding values expected in Type Ia supernova (SN Ia) enrichment are dramatically lower (Nagataki \& Sato 1998). We used our results to explore the origin of the metals in the Antennae. We calculated the $[\mathrm{Ne} / \mathrm{Fe}],[\mathrm{Mg} / \mathrm{Fe}]$, and $[\mathrm{Si} / \mathrm{Fe}]$ ratios of the regions listed in Table 1 and plotted them in diagrams displaying the $[\mathrm{Ne} / \mathrm{Fe}]$ ratio versus $[\mathrm{Mg} / \mathrm{Fe}]$ ratio (Fig. 4a), [Ne/Fe] versus [Si/Fe] (Fig. 4b), and $[\mathrm{Mg} / \mathrm{Fe}]$ versus $[\mathrm{Si} / \mathrm{Fe}](\mathrm{Fig} .4 c)$. In these diagrams, we also show the theoretical stellar yields expected from SNe Ia (red symbols) and SNe II (green symbols) taken from the compilations by Finoguenov et al. (2000) and Nagataki \& Sato (1998). We also indicate the regions occupied by the Type II supernova remnants (SNRs) Cas A and N132D, using the abundances measured by Favata et al. (1997a, 1997b) from BeppoSAX data. To relate our results to other measurements of these ratios in the literature, we plot also the values relative to the warm Galactic 

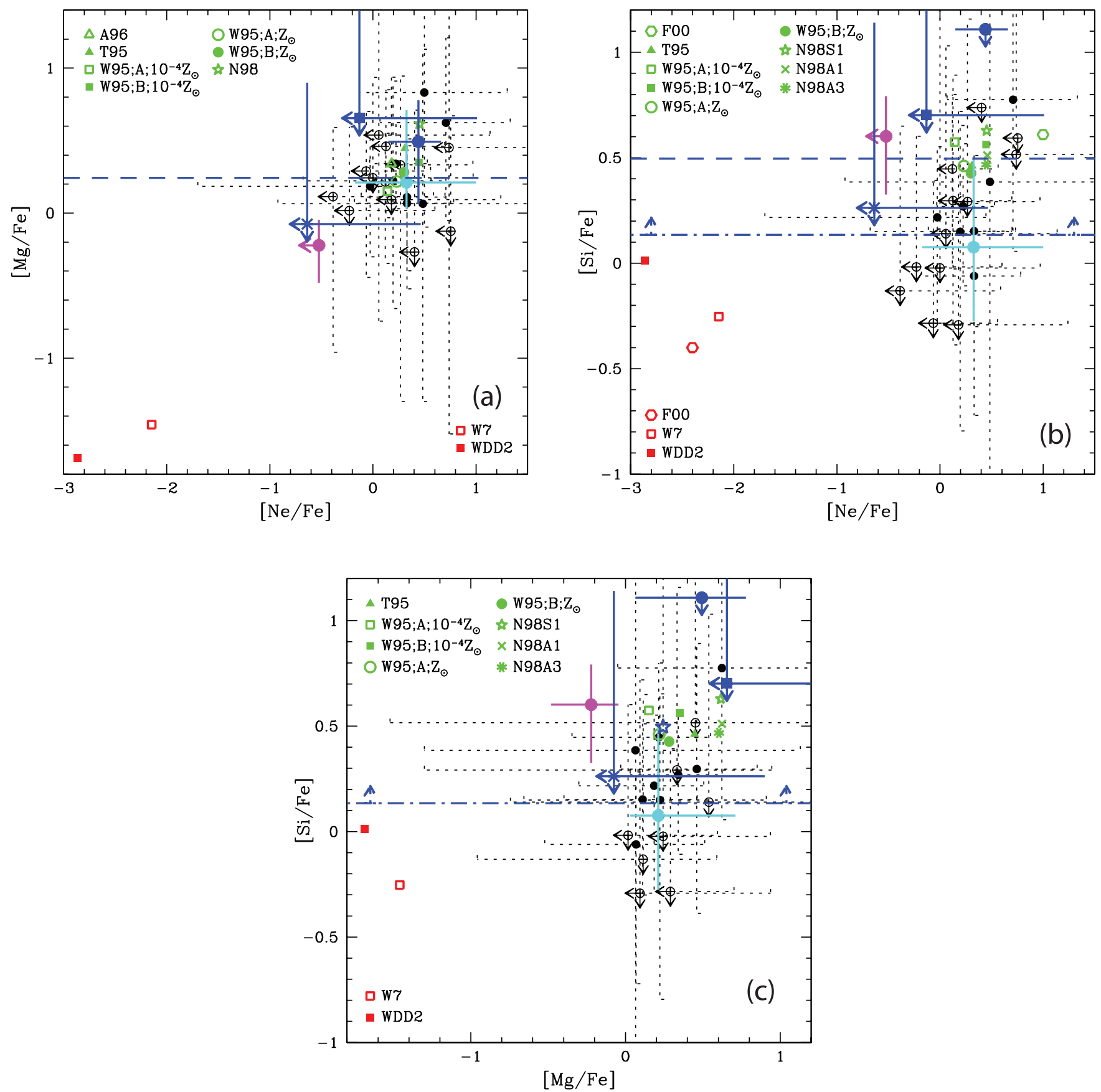

FIG. 4.-Abundance ratio diagrams for regions of the Antennae hot ISM: $(a)[\mathrm{Ne} / \mathrm{Fe}]$ vs. $[\mathrm{Mg} / \mathrm{Fe}] ;(b)[\mathrm{Ne} / \mathrm{Fe}]$ vs. $[\mathrm{Si} / \mathrm{Fe}] ;(c)[\mathrm{Mg} / \mathrm{Fe}]$ vs. [Si/Fe]. Abundance units refer to meteoritic abundances of Anders \& Grevesse (1989). The errors are at 68\%, and brackets represent the logarithmic values. Each one of the regions in Table 1 is plotted with a black circle, using an open circle if the detection significance of the $\mathrm{Mg}$ or Si line in the X-ray spectrum is less than $2 \sigma$. Upper limits are plotted with black arrows. Red symbols correspond to the ratios expected if all the elements were generated exclusively by SNe Ia, while green symbols refer to the average stellar yield of SNe II. "F00" indicates values adopted in Finoguenov et al. (2000), while the other labels refer to the theoretical works listed in Nagataki \& Sato (1998) and in Table 7. The purple and cyan symbols indicate abundance ratios for Cas A and N132D derived from the metallicities observed by BeppoSAX (Favata et al. 1997a, 1997b). Blue symbols and lines refer to determinations of abundance ratios in other galaxies: the warm Galactic halo (star symbol or dashed line), NGC 253 (northern halo, filled square; disk emission, cross), NGC 6946 (lower limit on [Si/Fe], dash-dotted line), and the Strickland et al. (2004) sample of edge-on starbursts ( filled circle).

halo (Savage \& Sembach 1996), to the emission both from the disk and from the northern halo of NGC 253 (Strickland et al. 2002), to the X-ray diffuse emission observed in NGC 6946 (Schlegel et al. 2003), and to the averaged value in the hot halo of a sample of 10 nearby edge-on starburst galaxies (Strickland et al. 2004).

Although the uncertainties are often large, almost all regions observed in the Antennae show $[\mathrm{Ne} / \mathrm{Fe}]$ and $[\mathrm{Mg} / \mathrm{Fe}]$ ratios fully consistent with a SN II-dominated enrichment scenario.
However, the $[\mathrm{Si} / \mathrm{Fe}]$ ratio is clearly lower than expected in a SN II enrichment scenario, and in some cases it could be consistent with SN Ia enrichment, although the uncertainties are quite large also for this ratio. The only two exceptions to these trends are regions 5 and 7 , both located in the upper right corners of the diagrams. These two regions have a $[\mathrm{Si} / \mathrm{Fe}]$ ratio fully consistent with an enrichment stemming entirely from Type II supernovae. Region 7 is the "overlap region" of the two merging galaxies, where the most active star formation is presently occurring 
(Mirabel et al. 1998; Wilson et al. 2000; Zhang et al. 2001). It seems plausible that recent episodes of star formation led to the injection of hot enriched gas into the ISM almost exclusively through SNe II. Region 5 is the region with the highest abundances of both $\mathrm{Mg}$ and Si. Similarly to region 7, region 5 has a high relative star formation strength and star formation rate per unit area (see Table 6 and discussion below).

There are five regions with very low $[\mathrm{Si} / \mathrm{Fe}]$ values located in the lower parts of Figures $4 b$ and $4 c([\mathrm{Si} / \mathrm{Fe}]<0$, even encompassing the confidence range). These low $[\mathrm{Si} / \mathrm{Fe}]$ values appear to be inconsistent with SN II enrichment and are more typical of SN Ia enrichment. We caution that this surprising result may be affected by poor statistics. Typically, in these regions the Si line is detected at less than $2 \sigma$ significance level, in some cases because of the poor statistics at $E \gtrsim 2 \mathrm{keV}$ that may lead to an underestimation of the Si abundance. However, note that this deficiency may be an independent confirmation of the failure of Type II SN yield predictions to fit the spectra (e.g., linking together all the $\alpha$-elements using the Type II SNe ratio from the compilation of average stellar yields from Nagataki \& Sato 1998; see Paper I).

While there may be some uncertainties both in the SN yield models and (even more) in our abundance measures, we may also be seeing the effect of different mean ages of star formation regions and, therefore, of different distributions of SN masses in different X-ray emission regions. This observed Si deficiency may also be explained if a significant fraction of the $\mathrm{Si}$, larger than for the other elements, has cooled and is locked in dust grains. Silicon is one of the most refractory elements, while neon is one of the least refractory elements, as indicated by depletions derived from interstellar absorption lines (see Savage \& Sembach 1996 for a review of this topic). However, iron is depleted at least as strongly as silicon, and there is no supporting observational or theoretical evidence to claim that silicon should be more strongly affected than iron by cooling and locking up in dust.

We note that the abundance ratios measured in the majority of the NGC 4038/4039 regions are consistent with ratios observed in SNR N132D. On the other hand, the measured ratios do not agree with the values observed in Cas A. The different measured ratios in these two SNRs, both originating from a Type II SN event, can give us an idea of the complexity and wide variety of input physics involved in yield predictions (e.g., convection, reaction rates, method for initiating shock waves; see Gibson et al. 1997).

Although our measurements are affected by large uncertainties, they represent clearly an improvement over the previous efforts aimed at measuring the $\alpha$-element-to-Fe ratio in starburst galaxies. The only exception comes from the Strickland et al. (2004) sample of edge-on starburst galaxies. This data point, which has smaller error bars than our typical error bars, is consistent with the ratios observed in almost all the regions of the Antennae. However, note that their smaller error bars derive from the fact that they come from the observed hot halo emission in all 10 galaxies of the sample. Moreover, in this sample they cannot put a stringent upper limit on the $[\mathrm{Si} / \mathrm{Fe}] \mathrm{ratio}$, a thing that we were able to do in at least some of our regions.

\subsection{X-Ray Abundances versus Optical/Radio SF Indicators}

To further explore our results, we searched for correlations with age and star formation indicators for the different regions of the Antennae. NGC 4038/4039 was observed by the Hubble Space Telescope Wide Field Planetary Camera 2 (HST WFPC2; F336W, F439W, F555W, and F814W filters) in 1996 January (Whitmore et al. 1999). There were also Very Large Array (VLA) observations (in configurations $\mathrm{BnA}, \mathrm{CnB}$, and $\mathrm{B}$ at 6 and $4 \mathrm{~cm}$ ) performed in 1997 January and 1998 September (Neff \& Ulvestad 2000). On the basis of the HST WFPC2 data and VLA observations, we looked for five different types of objects within each of our regions, counting (1) the number $R$ of very red, extremely young clusters (Zhang et al. 2001; 84 in total), (2) the number $B$ of bright young blue clusters (Table 1 in Whitmore et al. 1999; 50 in total), (3) the number $I$ of intermediate-age clusters (Table 2 in Whitmore et al. 1999; 30 in total, of which 5 are unpublished), (4) the number of flat-spectrum, thermal radio sources $(\alpha>$ -0.40 ), and (5) the number of steep-spectrum, nonthermal radio sources $(\alpha \leq-0.40)$. In addition, brightness estimates were performed visually on the HST WFPC2 images, aimed at estimating the integrated luminosity of each region in the $U, V, I$, and $\mathrm{H} \alpha$ passbands on a scale ranging from 0 to 10 .

From the published data and our visual estimates it was possible to derive for each region four quantities that might correlate with ISM metal abundances: a mean cluster age, the relative strength of star formation, the star formation per unit area, and the number of SNRs per unit area. The mean cluster age in each region was computed as the number-weighted average of the mean ages of very red clusters (4 Myr) and young blue clusters (15 Myr); we ignored the intermediate-age ( $\sim 500 \mathrm{Myr})$ clusters because their small known numbers $(0-2$ per region) would have added excessive noise. The relative strength of star formation was estimated via the ratio $(U+\mathrm{H} \alpha) /(V+I)$, where "relative" is with respect to the older populations contributing light to the $V$ and $I$ passbands. The star formation per unit area was calculated as the sum of $U$ and $\mathrm{H} \alpha$ estimates (on a scale of $0-10$ ) divided by the estimated area of each region in $\mathrm{kpc}^{2}$. The number of SNRs per unit area was defined as the number of VLA steep-spectrum radio sources (from Neff \& Ulvestad 2000) divided by the estimated area of the region.

Table 5 summarizes our visual estimates, while Table 6 is a summary of the derived quantities and also includes information about the presence of major star-forming complexes named by Rubin et al. (1970) in its last two columns. Figure 5 displays the relations between the four star formation indicators listed above and the mean abundance of $\alpha$-elements in each region. The mean abundance was calculated as the straight average of the $\mathrm{Ne}, \mathrm{Mg}$, and $\mathrm{Si}$ abundances, while the error bars were computed through the propagation of errors (at $1 \sigma$ ). As Figure 5 shows, there are no strong correlations between the different star formation indicators we calculated and the mean abundances in the same regions. The star formation per unit area and the number of SNRs per unit area are supposed to be among the best indicators of star formation. Yet these two quantities appear uncorrelated with the metal abundances, while a slight correlation may exist between the relative strength of star formation and the abundance of $\alpha$-elements. Figure 6 compares the same star formation indicators with $\mathrm{Fe}$ abundance. No obvious trend between the several quantities is detected in this case either, although a larger spread in $\mathrm{Fe}$ abundances seems to characterize regions with higher mean stellar ages.

The hot gas masses derived from abundances in $\S 3$ may yield some clues toward an interpretation of this lack of correlations. Assuming the average SN II stellar yields listed in Nagataki \& Sato (1998), we estimated the number of Type II SNe necessary to produce the observed metal masses in the hot ISM and the time required to produce that number of $\mathrm{SNe}$. If the resulting time is significantly shorter than $100 \mathrm{Myr}$, then the lack of correlations may either be related to the speed at which mixing occurs in the hot ISM or indicate that the metals are somehow removed from the hot phase of the ISM. Conversely, if the SN production 
TABLE 5

Mean Optical and Radio Observed Properties of the 17 Chandra Regions in NGC 4038/4039

\begin{tabular}{|c|c|c|c|c|c|c|c|c|c|}
\hline Region & $\begin{array}{c}H S T \\
\text { WFPC2 } R\end{array}$ & $\begin{array}{c}H S T \\
\text { WFPC2 } B\end{array}$ & $\begin{array}{c}H S T \\
\text { WFPC2 } I\end{array}$ & VLA Flat & VLA Steep & $\begin{array}{l}\text { Phot. } \\
H S T U\end{array}$ & $\begin{array}{l}\text { Phot. } \\
H S T V\end{array}$ & $\begin{array}{l}\text { Phot. } \\
\text { HST I }\end{array}$ & $\begin{array}{c}\text { Phot. } \\
H S T \mathrm{H} \alpha\end{array}$ \\
\hline $1^{\mathrm{a}} \ldots \ldots$ & 1 & 0 & 0 & 0 & 1 & 1 & 2 & 3 & 1 \\
\hline ............... & 1 & 3 & 0 & 0.5 & 5 & 9 & 5 & 4 & 7 \\
\hline З & 2 & 0 & 0 & 0 & 2 & 0 & 2 & 4 & 1 \\
\hline$\ldots \ldots \ldots \ldots \ldots$ & 1 & 2 & 2 & 0 & 0 & 1 & 3 & 6 & 2 \\
\hline 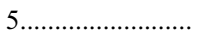 & 2 & 3 & 0 & 2 & 1 & 6 & 3 & 3 & 8 \\
\hline 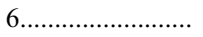 & 5 & 6 & 2 & 0 & 3 & 4 & 2 & 3 & 4 \\
\hline 7........................ & 16 & 0 & 0 & 3 & 11 & 0 & 1 & 3 & 4 \\
\hline $8 \ldots \ldots \ldots \ldots \ldots \ldots \ldots \ldots$ & 2 & 1 & 0 & 1 & 2 & 3 & 6 & 10 & 10 \\
\hline $9 \ldots \ldots \ldots \ldots \ldots \ldots \ldots \ldots \ldots \ldots \ldots \ldots \ldots$ & 0 & 7 & 0 & 3 & 4 & 3 & 4 & 5 & 10 \\
\hline 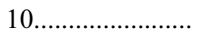 & 0 & 0 & 1 & 0 & 1 & 0 & 0 & 0 & 1 \\
\hline $11 \ldots \ldots \ldots \ldots \ldots \ldots \ldots$ & 4 & 3 & 2 & 1 & 5 & 6 & 3 & 3 & 8 \\
\hline $12 \ldots \ldots \ldots \ldots \ldots \ldots \ldots$ & 2 & 6 & 1 & 1 & 4 & 10 & 6 & 5 & 10 \\
\hline $13 \ldots \ldots \ldots \ldots \ldots \ldots \ldots$ & 0 & 3 & 0 & 2 & 0 & 6 & 5 & 5 & 8 \\
\hline 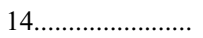 & 1 & 0 & 0 & 0 & 1 & 1 & 6 & 10 & 5 \\
\hline $15 \ldots \ldots \ldots \ldots \ldots \ldots \ldots$ & 0 & 2 & 0 & 0 & 2 & 3 & 3 & 8 & 6 \\
\hline $16 \ldots \ldots \ldots \ldots \ldots \ldots \ldots \ldots$ & 2 & 2 & 0 & 1 & 2 & 2 & 3 & 5 & 3 \\
\hline $17 \ldots \ldots \ldots \ldots \ldots \ldots \ldots$ & 10 & 2 & 1 & 3 & 15 & 0 & 2 & 3 & 1 \\
\hline
\end{tabular}

${ }^{\text {a }}$ Values for Region 1, based only on the eastern part (the western part was covered by WFPC2 to $<50 \%$ ).

time is $\gtrsim 100 \mathrm{Myr}$, then mean cluster ages may not be relevant because over periods of 100 Myr or longer, major fractions of the decaying galaxy orbits are traversed, and it is unclear whether, even if we were able to derive better local mean ages (i.e., including also intermediate-age clusters), any relation could be established between regional abundances and the past local star formation history.

Table 7 lists the results of our calculations for a range of theoretical models for SN II average yields (references are given in col. [1]). Columns (2), (3), (4), and (5) give the numbers of Type II SNe necessary to produce the observed masses of $\mathrm{Ne}$, $\mathrm{Mg}, \mathrm{Si}$, and $\mathrm{Fe}$, respectively. From these numbers, we estimated the time necessary to produce the required number of SNe II, assuming a constant SN rate throughout the Antennae. Neff \& Ulvestad (2000) derived a SN rate directly associated with the compact radio sources detected in the Antennae of $0.03 \mathrm{yr}^{-1}$, assuming that a typical SNR emits $6 \mathrm{~cm}$ radio emission for $30,000 \mathrm{yr}$ and has the luminosity of Cas A. However, applying the Condon \& Yin (1990) method (based on the ratio of nonthermal radio luminosity to the radio-emitting SN rate in our Galaxy) to the total steep-spectrum radio emission of the Antennae, and not only to the detected compact sources, they predict a SN rate of $0.26 \mathrm{yr}^{-1}$. The resulting times required to produce the quantity of $\mathrm{Ne}, \mathrm{Mg}, \mathrm{Si}$, and Fe observed in the Antennae through SN II enrichment were calculated for both of these estimates of the SN rate and are listed in columns (6), (7), (8), and (9), respectively. For both rate estimates the times required are short: in the $\sim 3.4-$ $12 \mathrm{Myr}$ range for a SN rate of $0.03 \mathrm{yr}^{-1}$ and in the $\sim 0.4-1.4 \mathrm{Myr}$ range for $0.26 \mathrm{yr}^{-1}$. Note that the second estimate is more reliable because the SN rate was derived by taking into account not

TABLE 6

Mean Optical and Radio Derived Properties of the 17 Chandra Regions in NGC 4038/4039

\begin{tabular}{|c|c|c|c|c|c|c|c|}
\hline Region & $\begin{array}{c}\text { Mean Age } \\
\text { (Myr) }\end{array}$ & $\begin{array}{c}\text { Relative SF } \\
\text { Strength }\end{array}$ & SF per Unit Area & SNRs per Unit Area & $\begin{array}{l}\text { Unit Area } \\
\left(\mathrm{kpc}^{2}\right)\end{array}$ & Galaxy $^{\mathrm{a}}$ & Landmark Objects $^{\mathrm{a}}$ \\
\hline $1^{\mathrm{b}} \ldots$ & 4.0 & 0.4 & 1.10 & 0.55 & 1.81 & 4038 & $\mathrm{~N}$ in "W Loop" \\
\hline $2 \ldots \ldots \ldots \ldots \ldots$ & 12.2 & 1.8 & 27.6 & 8.6 & 0.58 & 4038 & "G" complex \\
\hline $3 \ldots \ldots \ldots \ldots \ldots$ & 4.0 & 0.2 & 0.31 & 0.63 & 3.2 & 4038 & Nearly pure old disk \\
\hline $4 \ldots \ldots \ldots \ldots \ldots$ & 11.3 & 0.3 & 1.14 & 0.0 & 2.64 & 4038 & "H" and outer bulge, dust lane \\
\hline $5 \ldots \ldots \ldots \ldots \ldots$ & 10.6 & 2.3 & 29.2 & 2.1 & 0.48 & 4038 & $2 / 3$ "F," disk \\
\hline $6 \ldots \ldots \ldots \ldots \ldots$ & 10.0 & 1.6 & 2.1 & 0.8 & 3.8 & 4038 & 2/3 "E," strong SF in NE disk \\
\hline $7 \ldots \ldots \ldots \ldots \ldots$ & 4.0 & 1.0 & 1.7 & 4.6 & 2.4 & $\ldots$ & "Overlap region" \\
\hline $8 \ldots \ldots \ldots \ldots \ldots$ & 7.7 & 0.8 & 8.9 & 1.4 & 1.46 & 4039 & "A," nucleus and bulge of 4039 \\
\hline $9 \ldots \ldots \ldots \ldots \ldots$ & 15.0 & 1.4 & 3.2 & 1.0 & 4.0 & 4039 & "B" and "AA," outer bulge of 4039 \\
\hline $10 \ldots \ldots \ldots \ldots$ & $\ldots$ & $\ldots$ & 0.13 & 0.13 & 7.8 & $\ldots$ & Interdisk region, mostly empty \\
\hline $11 \ldots \ldots \ldots \ldots . . .$. & 8.7 & 2.3 & 10.8 & 3.8 & 1.3 & 4038 & "M," S in "W Loop" \\
\hline $12 \ldots \ldots \ldots \ldots \ldots$ & 12.2 & 1.8 & 29.0 & 5.7 & 0.70 & 4038 & "S" and "T," in "W Loop" \\
\hline $13 \ldots \ldots \ldots \ldots$ & 15.0 & 1.4 & 56.0 & 0.0 & 0.25 & 4038 & "R," in "W Loop" \\
\hline $14 \ldots \ldots \ldots \ldots$ & 4.0 & 0.4 & 32.0 & 5.3 & 0.19 & 4038 & "J," $\mathrm{N}$ part of inner bulge \\
\hline $15 \ldots \ldots \ldots \ldots$ & 15.0 & 0.8 & 39.0 & 8.7 & 0.23 & 4038 & "K," S part of inner bulge and nucleus of 4038 \\
\hline $16 \ldots \ldots \ldots \ldots$ & 9.5 & 0.6 & 1.6 & 0.65 & 3.1 & 4038 & "L," outer bulge of 4038 \\
\hline $17 \ldots \ldots \ldots \ldots \ldots$ & 5.8 & 0.2 & 0.22 & 3.3 & 4.5 & $\ldots$ & "Overlap region," most dusty part \\
\hline
\end{tabular}

a Location from Rubin et al. (1970).

b Values for Region 1, based only on the eastern part (the western part was covered by WFPC2 to $<50 \%$ ). 

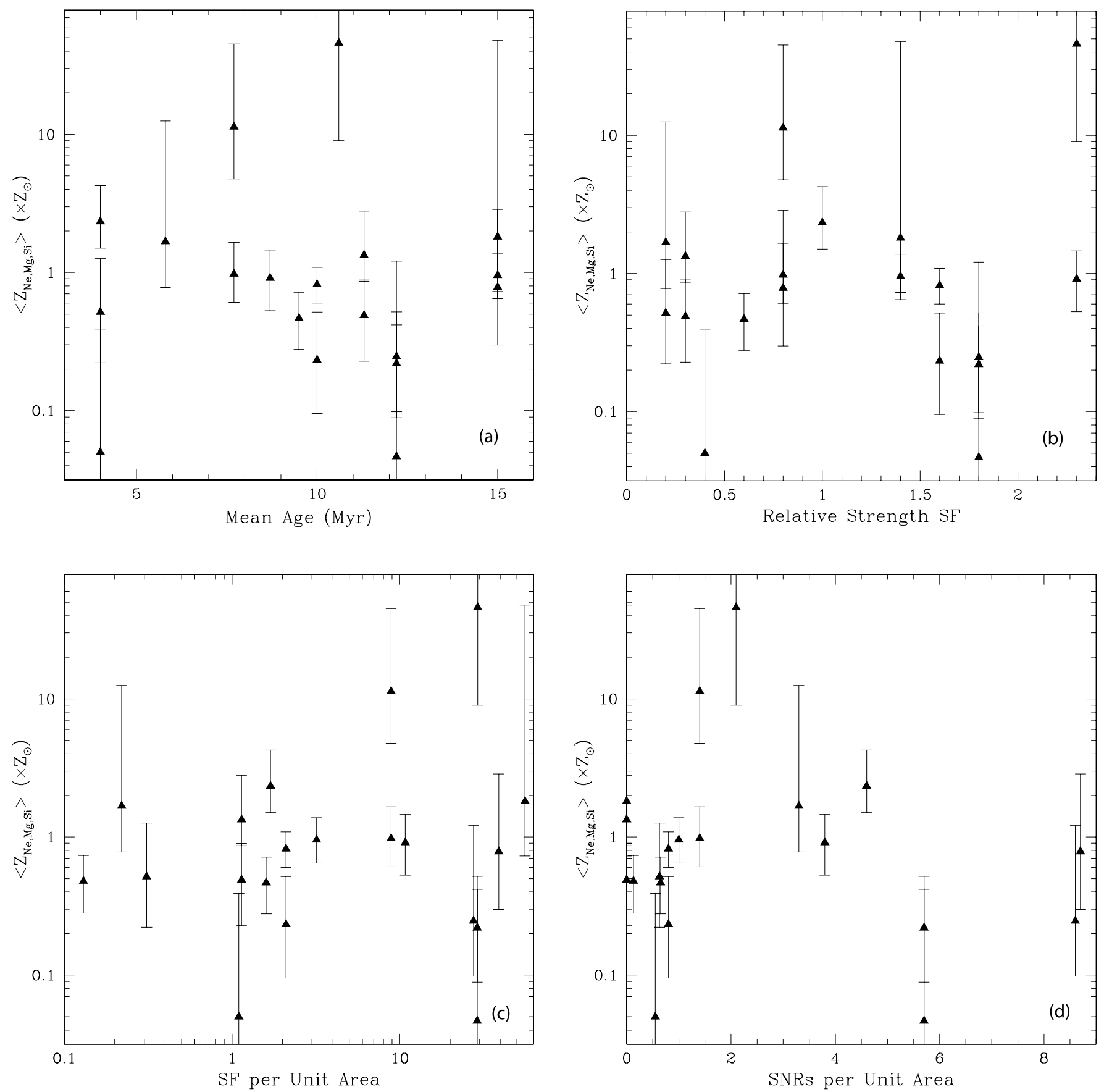

FIG. 5.-Diagrams of the mean abundances of $\alpha$-elements in each region of the Antennae hot ISM plotted vs. $(a)$ mean age of the star clusters, $(b)$ relative strength of star formation, $(c)$ star formation per unit area, and $(d)$ SNRs per unit area. 

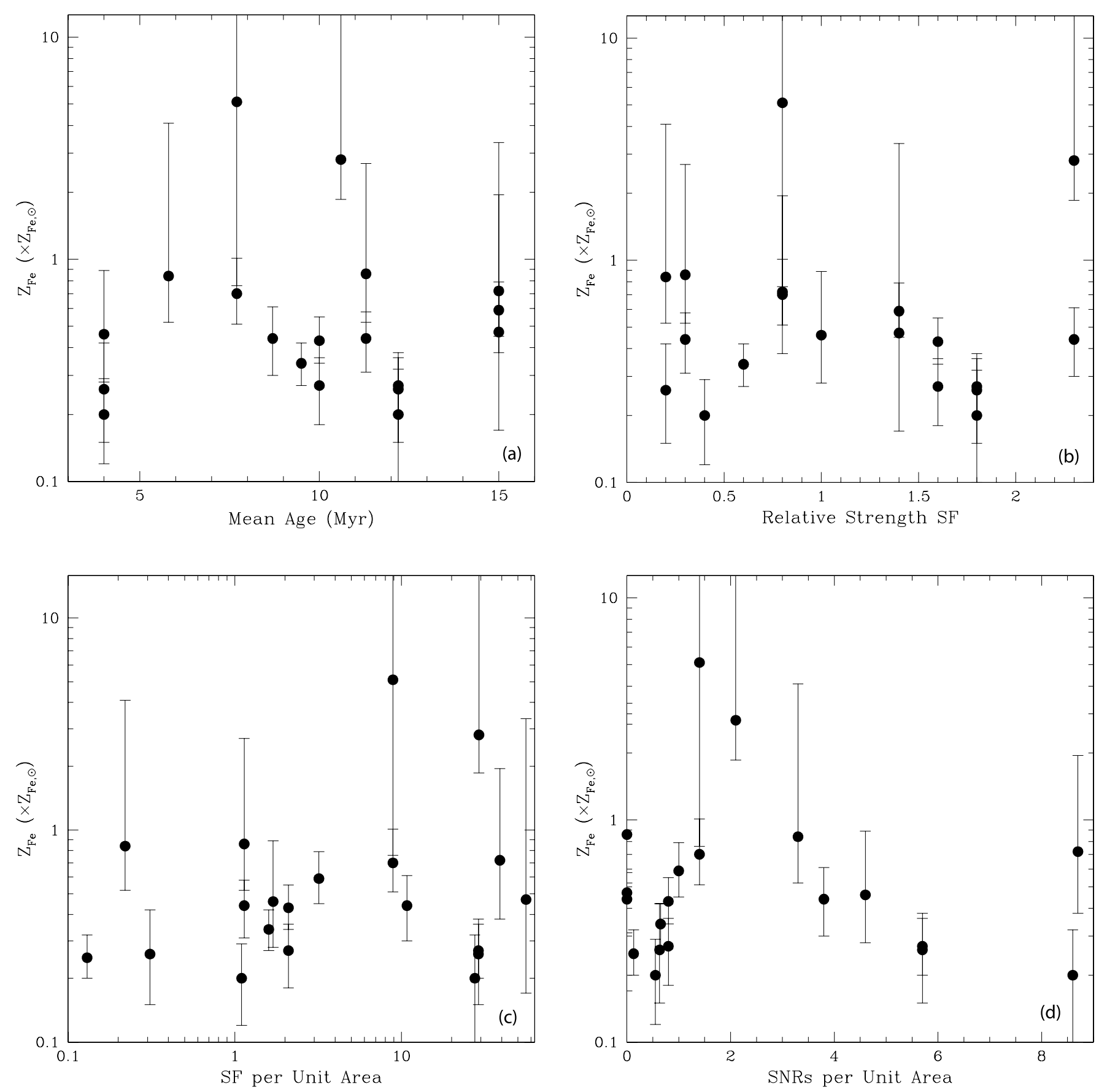

Fig. 6.-Diagrams of the Fe abundance in each region of the Antennae hot ISM plotted vs. (a) mean age of the star clusters, $(b)$ relative strength of star formation, (c) star formation per unit area, and (d) SNRs per unit area. 
TABLE 7

Number of Type II SNe and Time Required to Produce the Observed Metal Masses

\begin{tabular}{|c|c|c|c|c|c|c|c|c|}
\hline $\begin{array}{c}\text { Reference }^{\mathrm{a}} \\
\text { (1) }\end{array}$ & $\begin{array}{c}N_{\mathrm{SN} \text { II }}(\mathrm{Ne}) \\
\left(10^{5}\right) \\
(2)\end{array}$ & $\begin{array}{c}N_{\mathrm{SN} \text { II }}(\mathrm{Mg}) \\
\left(10^{5}\right) \\
(3)\end{array}$ & $\begin{array}{c}N_{\mathrm{SN} \text { II }}(\mathrm{Si}) \\
\left(10^{5}\right) \\
(4)\end{array}$ & $\begin{array}{c}N_{\mathrm{SN} \mathrm{II}}(\mathrm{Fe}) \\
\left(10^{5}\right) \\
(5)\end{array}$ & $\begin{array}{c}t_{\mathrm{SN} \mathrm{II}}(\mathrm{Ne})^{\mathrm{b}} \\
(\mathrm{Myr}) \\
(6)\end{array}$ & $\begin{array}{c}t_{\mathrm{SN} \text { II }}(\mathrm{Mg})^{\mathrm{b}} \\
(\mathrm{Myr}) \\
(7)\end{array}$ & $\begin{array}{c}t_{\mathrm{SN} \mathrm{II}}(\mathrm{Si})^{\mathrm{b}} \\
(\mathrm{Myr}) \\
(8)\end{array}$ & $\begin{array}{c}t_{\mathrm{SN} \mathrm{II}}(\mathrm{Fe})^{\mathrm{b}} \\
(\mathrm{Myr}) \\
(9)\end{array}$ \\
\hline 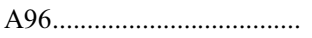 & 3.4 & 2.4 & $\ldots$ & 2.5 & $11.3,1.3$ & $8.0,0.9$ & $\ldots$ & $8.4,1.0$ \\
\hline 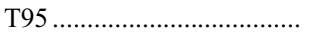 & 1.5 & 1.1 & 2.0 & 1.5 & $4.9,0.6$ & $3.7,0.4$ & $6.6,0.8$ & $4.9,0.6$ \\
\hline 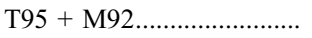 & $\ldots$ & $\ldots$ & $\ldots$ & 1.5 & $\ldots$ & $\ldots$ & $\ldots$ & $4.9,0.6$ \\
\hline W95; A; $10^{-4} Z_{\odot} \ldots \ldots \ldots \ldots . . . . .$. & 3.6 & 3.6 & 2.5 & 2.5 & $12.0,1.4$ & $12.0,1.4$ & $8.4,1.0$ & $8.2,0.9$ \\
\hline W95; B; $10^{-4} Z_{\odot} \ldots \ldots \ldots \ldots \ldots$. & 1.5 & 2.0 & 2.2 & 2.1 & $5.1,0.6$ & $6.6,0.8$ & $7.4,0.9$ & $7.0,0.8$ \\
\hline 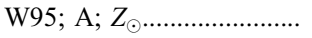 & 1.9 & 2.0 & 2.1 & 1.6 & $6.3,0.7$ & $6.7,0.8$ & $7.0,0.8$ & $5.3,0.6$ \\
\hline 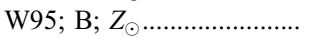 & 1.3 & 1.4 & 1.8 & 1.3 & $4.3,0.5$ & $4.6,0.5$ & $6.1,0.7$ & $4.2,0.5$ \\
\hline 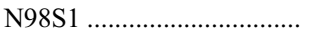 & 1.5 & 1.1 & 1.9 & 2.1 & $5.0,0.6$ & $3.5,0.4$ & $6.3,0.7$ & $6.9,0.8$ \\
\hline 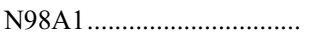 & 1.5 & 1.0 & 2.5 & 2.1 & $4.9,0.6$ & $3.4,0.4$ & $8.2,1.0$ & $6.9,0.8$ \\
\hline 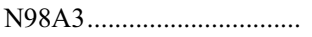 & 1.5 & 1.1 & 2.7 & 2.0 & $4.9,0.6$ & $3.5,0.4$ & $8.9,1.0$ & $6.8,0.8$ \\
\hline
\end{tabular}

a (A96) Arnett 1996; (M92) Maeder 1992; (N98) Nagataki et al. 1998; (T95) Tsujimoto et al. 1995; (W95) Woosley \& Weaver 1995. N98S1, N98A1, and

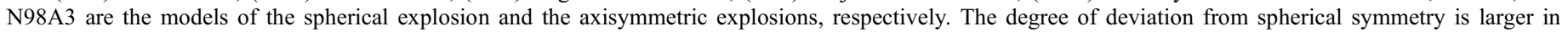
N98A3 than in N98A1.

${ }^{\mathrm{b}}$ Computed for a SN rate of 0.03 and $0.26 \mathrm{yr}^{-1}$, respectively (see text).

only the radio emission from the identifiable compact sources, but also the overall steep-spectrum radio emission from the whole galaxy.

However, if the average density of the ISM is lower than our estimates (e.g., if the disk thickness is larger than our assumed $200 \mathrm{pc}$ ), so that more mass and more metals are needed to produce the observed line intensities, then the above times may increase to $\sim 3-4 \mathrm{Myr}$ (or $\sim 25-35 \mathrm{Myr}$ in the lower SN rate estimate). Such time spans may be too short for the metals to become mixed throughout the hot ISM. Note also that a filling factor of the hot gas lower than 1 would increase the density estimate and make such time spans shorter.

Indeed, 2 Myr is a relevant time threshold for mixing in a hot gas of the temperature we detect, corresponding to average velocities of $\sim 500 \mathrm{~km} \mathrm{~s}^{-1}$. At $500 \mathrm{~km} \mathrm{~s}^{-1}$, gas travels only $\sim 1 \mathrm{kpc}$ in 2 Myr. If the time required to produce the metals is actually on the order of $\lesssim 2 \mathrm{Myr}$, it may be unlikely that efficient mixing takes place on a scale larger than a few hundred pc, and it is probably insufficient to destroy the correlation. Other factors may have destroyed the correlation as well, such as, for example, (1) the presence of a significant fraction of the SN ejecta in a cool phase or (2) in dust grains, or (3) the escape of the ejecta in a galactic wind.

Another possibility could be that the other metals are invisible in soft X-rays. Indeed, in a Chevalier \& Clegg (1985) wind model, the metals would be expected to be in much hotter $\left(T \sim 10^{7.5} \mathrm{~K}\right)$ gas. Therefore, the hot gas observed could be primarily ambient ISM heated, but not particularly enriched, by Type II SNe.

\section{PHYSICAL PROPERTIES OF THE HOT ISM}

The overall characteristics of the hot, diffuse gas in the Antennae, as noted by Read et al. (1995) and F03, are (1) a very high pressure, ranging from 10 to 100 times the pressure of the hot component of the ISM in the solar neighborhood (Cox \& Reynolds 1987), (2) temperatures in the range $0.3-0.7 \mathrm{keV}$, or about 3-7 times the temperature of the Galactic hot ISM, and (3) an abundance of $\alpha$-process elements generally consistent with the $\sim 0.5$ solar abundance shown by the stellar populations (Fritze-v. Alvensleben 1998).

There are, of course, uncertainties both in the model fits and in the path length that must be assumed to convert emission measures to pressures. In $\S 3$ we assumed a 200 pc thickness for the emitting region and a filling factor of 1 , unless explicit factors are given. Where two temperatures are required, we assumed the components to be in pressure equilibrium and their combined path length to be $200 \mathrm{pc}$.

In the spectral analysis above we have pinned down the metallicity parameters for specific regions, and we find abundances of $\mathrm{Ne}, \mathrm{Mg}$, and $\mathrm{Si}$ generally ranging from $\sim 0.2 Z_{\odot}$ to $Z_{\odot}$, along with a few cases of overabundance, represented mainly by five regions: $4 \mathrm{~b}, 5,7,8 \mathrm{a}$, and $8 \mathrm{~b}$.

Three of these regions can be defined as "hot diffuse emission regions" (regions 4b, 7, and 8a), requiring fitting either with two temperatures, one of which has to be consistently above $0.7 \mathrm{keV}$, or with a single temperature above $0.5 \mathrm{keV}$. On the other hand, the "cooler diffuse emission regions" (regions 5 and $8 \mathrm{~b}$ ) are both well fitted with a single temperature around $0.3 \mathrm{keV}$.

\subsection{Thermal Energy Supply from Type II SNe}

The five regions overabundant in metals listed above represent a heterogeneous subsample of our X-ray diffuse emission regions.

Region $4 \mathrm{~b}$ lies in the X-ray ridge that runs from northwest to southeast. Together with regions $4 \mathrm{a}, 6 \mathrm{a}$, and $6 \mathrm{~b}$, it is bounded to the northwest by a strong $\mathrm{H}$ I arm and to the southeast by a region of strong $\mathrm{CO}$ emission. Star formation in this region is modest as judged by the number of young clusters (Whitmore et al. 1999), and radio continuum emission is correspondingly weak (Hibbard et al. 2001). The mean age of the clusters is about $10 \mathrm{Myr}$ (Whitmore \& Schweizer 1995). Thus, this region is relatively quiescent and presents a relatively simple case for analysis.

On the other hand, region 7 corresponds to the most active burst of star formation currently occurring in the Antennae, as observed both in the optical and in the near-infrared (Mirabel et al. 1998; Wilson et al. 2000; Zhang et al. 2001). It also corresponds to the overlap region between the two merging disks and is discussed in more detail by Fabbiano et al. (2004).

Regions $8 \mathrm{a}$ and $8 \mathrm{~b}$ are strongly influenced by the presence of the southern galactic nucleus and present, together with region 7 , the highest intrinsic absorptions measured in the various spectral fits.

Finally, region 5 stands out for its very high abundances and high concentration of young star clusters. It, too, is discussed by Fabbiano et al. (2004). Its high abundance of $\alpha$-elements 
TABLE 8

Thermal Energy Supplies for the Five Regions Presenting Overabundances of Metals

\begin{tabular}{|c|c|c|c|c|}
\hline $\begin{array}{l}\text { Region } \\
\text { (1) }\end{array}$ & $\begin{array}{c}E_{\text {th }}(\mathrm{Ne}) \\
\text { (ergs) } \\
\text { (2) }\end{array}$ & $\begin{array}{c}E_{\text {th }}(\mathrm{Mg}) \\
\text { (ergs) } \\
\text { (3) }\end{array}$ & $\begin{array}{c}E_{\text {th }}(\mathrm{Si}) \\
\text { (ergs) } \\
\text { (4) }\end{array}$ & $\begin{array}{c}E_{\mathrm{th}}(\mathrm{Fe}) \\
(\mathrm{ergs}) \\
(5)\end{array}$ \\
\hline $4 \mathrm{~b} \ldots . .$. & $1.02_{-0.74}^{+1.20} \times 10^{55}$ & $9.42_{-6.06}^{+28.44} \times 10^{54}$ & $5.41_{-3.69}^{+5.10} \times 10^{54}$ & $1.27_{-0.51}^{+2.78} \times 10^{55}$ \\
\hline 5 & $1.64_{-0.85}^{+2.51} \times 10^{55}$ & $3.06_{-2.49}^{+51.99} \times 10^{55}$ & $1.20_{-1.20}^{+5.62} \times 10^{56}$ & $1.02_{-0.35}^{+32.34} \times 10^{55}$ \\
\hline 7 & $1.72_{-1.46}^{+3.07} \times 10^{55}$ & $1.23_{-0.84}^{+2.11} \times 10^{55}$ & $1.19_{-0.77}^{+1.32} \times 10^{55}$ & $6.61_{-2.65}^{+6.32} \times 10^{54}$ \\
\hline 8а................................. & $1.30_{-0.82}^{+1.60} \times 10^{55}$ & $6.16_{-4.41}^{+7.34} \times 10^{54}$ & $2.50_{-2.28}^{+4.04} \times 10^{54}$ & $1.18_{-0.33}^{+0.54} \times 10^{55}$ \\
\hline $8 \mathrm{~b}$ & $5.61_{-5.27}^{+\infty .02} \times 10^{55}$ & $1.87_{-1.07}^{+46.04} \times 10^{55}$ & $2.66_{-2.59}^{+\infty} \times 10^{55}$ & $3.61_{-3.15}^{+4.01} \times 10^{55}$ \\
\hline
\end{tabular}

compared to iron is consistent with enrichment by Type II SNe. Its low temperature (see Table 1) could be the result of a relatively high local gas density or of relatively effective entrainment of cool ISM gas into the low-density SN-heated gas. It may evolve into a state similar to that of the neighboring regions 4 and 6 over the course of a few times $10^{7} \mathrm{yr}$.

Following a similar procedure to the one described in $\S 4$, we derived first the mass of each chemical element from the spectral fits and then the number of Type II SNe required to produce the observed quantity of $\mathrm{Ne}, \mathrm{Mg}, \mathrm{Si}$, and $\mathrm{Fe}$ in the five above regions. The required number of $\mathrm{SNe}$ can yield an estimate of the total supply of thermal energy available to each region. Our estimates were performed by averaging the numbers computed from each of the SN yield models considered in Table 7 and propagating the errors. Assuming an average energy supply of $10^{51}$ ergs per $\mathrm{SN}$, we then obtained the thermal energies listed in Table 8. For each region the thermal energy supply derived from the observed quantity of $\mathrm{Ne}, \mathrm{Mg}, \mathrm{Si}$, and $\mathrm{Fe}$ are shown in columns (2), (3), (4), and (5), respectively.

If we compare these numbers with the thermal energies derived from the spectral fits (listed in Table 3), we can see that the observed thermal energy is systematically lower than the energy supplied by SNe. Note that filling factors less than unity would not affect this difference, since both thermal energies and masses scale as $\eta^{1 / 2}$. This discrepancy is generally about a factor of 5, with the exceptions of regions 5 and $8 \mathrm{~b}$, where the observed thermal energy is lower by about 2 orders of magnitude. Although the large errors in the metallicities of region $8 \mathrm{~b}$ can make this discrepancy less dramatic (and comparable with the other three regions), region 5 does not present such large uncertainties and clearly stands out with respect to the other emission regions. It is likely that in region 5 most of the thermal energy of the SNe that enriched the hot gas ( $\sim 99 \%$ ) has been lost either through thermal conduction and radiative cooling or through a wind.

Radiative cooling of the hot gas can be clearly ruled out by the cooling times listed in Table 3, which are definitely too long. On the other hand, the conductive cooling timescale for a volume of size $L$, temperature $T$, and density $n$ is

$$
t_{\text {cond }}=\frac{5 n k T L^{3}}{10^{-5} T^{5 / 2}(T / L) L^{2}}=\left(9 \times 10^{8}\right) n T_{0.5}^{-5 / 2} L_{\mathrm{pc}}^{2} \mathrm{~s},
$$

where $T_{0.5}$ is the temperature in units of $0.5 \mathrm{keV}$ and $L_{\mathrm{pc}}$ is the length scale in pc. For $n \sim 0.05 \mathrm{~cm}^{-3}$ (the typical electron density we observe in the Antennae X-ray diffuse emission), a temperature of $0.5 \mathrm{keV}$, and a length scale of $L \sim 30 \mathrm{pc}$, this formula yields a conductive timescale of $\sim 1000 \mathrm{yr}$, assuming full Spitzer conductivity (Spitzer 1962). Thus, conduction may have been able to dissipate a significant fraction of the thermal energy produced by Type II SNe in region 5 and in the other regions we examined, if hot and cool gas are mixed on a scale comparable to that in the Galaxy.

Loss of energy by a wind seems to be a possibility, too. However, a wind is supposed to carry away metals as well. Therefore, one would require the energy loss to the wind to be $\sim 100$ times more efficient than the mass loss (at least for region 5). This would suggest a wind temperature $\sim 100$ times higher than the temperature of the gas we observe, which looks implausible.

\section{SUMMARY AND CONCLUSIONS}

In this paper and in Paper I we have performed an extensive study of the X-ray properties of the diffuse emission of the Antennae (NGC 4038/4039), analyzing the entire $411 \mathrm{ks}$ exposure obtained with Chandra ACIS-S. Confirming the results of F03, based on the first of the seven observations used in the present study, we report a spatially and spectrally complex hot ISM. Thanks to our deep data, we also detect clear, spatially variable emission lines of $\mathrm{Ne}, \mathrm{Mg}$, and $\mathrm{Si}$, in addition to the $\mathrm{Fe} \mathrm{L}$ blend, which indicate supersolar metal abundances in a few regions of the hot ISM. In this paper we have tried to investigate the origin of these metals.

To summarize:

1. We derived physical parameters of the hot gas, such as density, thermal energy, cooling times, and pressure. This hot gas has cooling times lying in the $10^{7}-10^{8} \mathrm{yr}$ range, in agreement with the results of F03. At least in one of the two nuclei the hot gas pressure is significantly higher than the CO pressure, implying that shock waves may be driven into the CO clouds. Summing the hot gas masses determined for each region yields a total measured mass of $\sim 1.2 \times 10^{7} M_{\odot}$ for the hot ISM.

2. By fitting the Fe L, Ne Ix, Mg XI, and Si XIII emission, we find significant metal enrichment of the hot ISM. Metal abundances are generally consistent with solar, but reach extremes of $\sim 20-30$ times solar in region 5 and are significantly subsolar in a few regions. The total hot gas masses derived for $\mathrm{Fe}, \mathrm{Ne}, \mathrm{Mg}$, and $\mathrm{Si}$ are $1.8 \times 10^{4}, 3.4 \times 10^{4}, 1.3 \times 10^{4}$, and $2.6 \times 10^{4} \mathrm{M}_{\odot}$, respectively.

3. Comparison of elemental ratios with those expected from Type Ia and Type II SNe suggests that SNe II are mainly responsible for the metal enrichment of the hot ISM in the Antennae. However, we measure $[\mathrm{Si} / \mathrm{Fe}]$ ratios mostly lower than those predicted for SN II yields. A similar inconsistency was also found in Paper I, linking together all the $\alpha$-elements using the Type II SNe ratio from the compilation of average stellar yields. Although there surely are considerable uncertainties in the SN yields predicted by theoretical models, it is also true that our spectra are affected by low statistics at the energy of the Si line $(E \sim 2 \mathrm{keV})$. This suggests that uncertainties in the measurements of the $\mathrm{Si}$ abundance may be the primary cause of this discrepancy. 
If our measurements are correct, our result may point to a depletion of Si in the hot ISM, perhaps from more efficient cooling of this element.

4. We report a remarkable lack of correlations between our abundance measurements and stellar age indicators estimated from either the HST observations of stellar clusters (Whitmore \& Schweizer 1995; Whitmore et al. 1999) or VLA SNR estimates (Neff \& Ulvestad 2000). The time required to produce the observed quantities of metals through Type II SN explosions is probably $\lesssim 2 \mathrm{Myr}$, implying that efficient mixing is unlikely to be the main agent destroying the expected correlation between abundances and radio-optical star formation indicators. This may point toward the presence of a significant fraction of SN II ejecta in a cool phase, in dust grains, or escaping in a wind of hot gas, undetectable at soft X-ray wavelengths.

5. We have analyzed the energy input coming from SN explosions in the five regions with metallicities significantly higher than the average stellar abundance $(\sim 0.5$ solar; Fritze $-v$. Alvensleben
1998). In all of these regions we observe a thermal energy lower than expected from their metallicities and $\mathrm{SN}$ enrichment. We conclude that the missing energy has been dissipated through processes different than radiative cooling (radiative $\tau_{c} \sim 10^{7}-$ $10^{8} \mathrm{yr}$ ), most likely through conductive cooling.

We thank the Chandra X-Ray Center Data Systems (DS) and Science Data Systems (SDS) teams for their efforts in reducing the data and for developing the software used in the data reduction (SDP) and analysis (CIAO). We thank D.-W. Kim for useful discussions. We also thank the anonymous referee for his careful analysis of the paper and for giving us helpful suggestions for the presentation of our results. This work was supported in part by NASA contract NAS8-39073 and NASA grants GO12115X and GO2-3135X. F. S. acknowledges partial support from the NSF through grant AST 02-05994.
Aguirre, A., Hernquist, L., Schaye, J., Weinberg, D. H., Katz, N., \& Gardner, J. 2001, ApJ, 560, 599

Anders, E., \& Grevesse, N. 1989, Geochim. Cosmochim. Acta, 53, 197

Arnett, D. 1996, Supernovae and Nucleosynthesis (Princeton: Princeton Univ. Press)

Baldi, A., Raymond, J. C., Fabbiano, G., Zezas, A., Rots, A. H., Schweizer, F., King, A. R., \& Ponman, T. J. 2006, ApJS, in press (Paper I)

Chevalier, R. A., \& Clegg, A. W. 1985, Nature, 317, 44

Chiang, W.-H., Ryu, D., \& Vishniac, E. T. 1988, PASP, 100, 1386

Condon, J. J., \& Yin, Q. F. 1990, ApJ, 357, 97

Cox, D. P., \& Reynolds, R. J. 1987, ARA\&A, 25, 303

Dahlem, M., Parmar, A., Oosterbroek, T., Orr, A., Weaver, K. A., \& Heckman, T. M. 2000, ApJ, 538, 555

Dahlem, M., Weaver, K. A., \& Heckman, T. M. 1998, ApJS, 118, 401

de Avillez, M. A., \& Breitschwerdt, D. 2004, A\&A, 425, 899

Fabbiano, G., Krauss, M., Zezas, A., Rots, A., \& Neff, S. 2003, ApJ, 598, 272 (F03)

Fabbiano, G., Schweizer, F., \& Mackie, G. 1997, ApJ, 478, 542

Fabbiano, G., \& Trinchieri, G. 1983, ApJ, 266, L5

Fabbiano, G., Zezas, A., \& Murray, S. S. 2001, ApJ, 554, 1035

Fabbiano, G., et al. 2004, ApJ, 605, L21

Favata, F., Vink, J., Parmar, A. N., Kaastra, J. S., \& Mineo, T. 1997a, A\&A, 324, L45

Favata, F., et al. 1997b, A\&A, 324, L49

Finoguenov, A., David, L. P., \& Ponman, T. J. 2000, ApJ, 544, 188

Finoguenov, A., Matsushita, K., Böhringer, H., Ikebe, Y., \& Arnaud, M. 2002, A\&A, 381, 21

Fritze-v. Alvensleben, U. 1998, A\&A, 336, 83

Gibson, B. K., Loewenstein, M., \& Mushotzky, R. F. 1997, MNRAS, 290, 623

Heckman, T. M. 1999, Ap\&SS, 266, 3

Hibbard, J. E., van der Hulst, J. M., Barnes, J. E., \& Rich, R. M. 2001, AJ, 122, 2969

Huo, Z. Y., Xia, X. Y., Xue, S. J., Mao, S., \& Deng, Z. G. 2004, ApJ, 611, 208

Maeder, A. 1992, A\&A, 264, 105 (erratum 268, 833 [1993])

Martin, C. L., Kobulnicky, H. A., \& Heckman, T. M. 2002, ApJ, 574, 663

McKee, C. F. 1995, in ASP Conf. Ser. 80, The Physics of the Interstellar Medium, ed. A. Ferrara, C. F. McKee, C. Heiles, \& P. R. Shapiro (San Francisco: ASP), 292

McKee, C. F., \& Ostriker, J. P. 1977, ApJ, 218, 148

Metz, J. M., Cooper, R. L., Guerrero, M. A., Chu, Y.-H., Chen, C.-H. R., \& Gruendl, R. A. 2004, ApJ, 605, 725

Mirabel, I. F., et al. 1998, A\&A, 333, L1

Mushotzky, R., Loewenstein, M., Arnaud, K. A., Tamura, T., Fukazawa, Y., Matsushita, K., Kikuchi, K., \& Hatsukade, I. 1996, ApJ, 466, 686
Nagataki, S., Hashimoto, M.-A., Sato, K., Yamada, S., \& Mochizuki, Y. S. 1998, ApJ, 492, L45

Nagataki, S., \& Sato, K. 1998, ApJ, 504, 629

Neff, S. G., \& Ulvestad, J. S. 2000, AJ, 120, 670

Norman, C. A., \& Ferrara, A. 1996, ApJ, 467, 280

Pudritz, R. E., \& Fiege, J. D. 1999, in ASP Conf. Ser. 168, New Perspectives on the Interstellar Medium, ed. A. R. Taylor, T. L. Landecker, \& G. Joncas (San Francisco: ASP), 235

Read, A. M., Ponman, T. J., \& Wolstencroft, R. D. 1995, MNRAS, 277, 397

Rubin, V. C., Ford, W. K., Jr., \& D’Odorico, S. 1970, ApJ, 160, 801

Sansom, A. E., Dotani, T., Okada, K., Yamashita, A., \& Fabbiano, G. 1996, MNRAS, 281, 48

Savage, B. D., \& Sembach, K. R. 1996, ARA\&A, 34, 279

Saviane, I., Hibbard, J. E., \& Rich, R. M. 2004, AJ, 127, 660

Schlegel, E. M., Holt, S. S., \& Petre, R. 2003, ApJ, 598, 982

Soria, R., \& Wu, K. 2003, A\&A, 410, 53

Spitzer, L. 1962, Physics of Fully Ionized Gases (2nd ed.; New York: Interscience)

Strickland, D. K., Heckman, T. M., Colbert, E. J. M., Hoopes, C. G., \& Weaver, K. A. 2004, ApJS, 151, 193

Strickland, D. K., Heckman, T. M., Weaver, K. A., Hoopes, C. G., \& Dahlem, M. 2002, ApJ, 568, 689

Strickland, D. K., \& Stevens, I. R. 1998, MNRAS, 297, 747

. 2000, MNRAS, 314, 511

Tsujimoto, T., Nomoto, K., Yoshii, Y., Hashimoto, M., Yanagida, S., \& Thielemann, F.-K. 1995, MNRAS, 277, 945

Tucker, W. H. 1975, Radiation Processes in Astrophysics (Cambridge: MIT Press)

Weaver, K. A., Heckman, T. M., \& Dahlem, M. 2000, ApJ, 534, 684

Weisskopf, M. C., O’Dell, S. L., \& van Speybroeck, L. P. 1996, Proc. SPIE, 2805, 2

Whitmore, B. C., \& Schweizer, F. 1995, AJ, 109, 960

Whitmore, B. C., Zhang, Q., Leitherer, C., Fall, S. M., Schweizer, F., \& Miller, B. W. 1999, AJ, 118, 1551

Wilson, C. D., Scoville, N., Madden, S. C., \& Charmandaris, V. 2000, ApJ, 542,120

Wiseman, J. J., \& Ho, P. T. P. 1998, ApJ, 502, 676

Woosley, S. E., \& Weaver, T. A. 1995, ApJS, 101, 181

Zezas, A., Fabbiano, G., Rots, A. H., \& Murray, S. S. 2002, ApJS, 142, 239

Zhang, Q., Fall, S. M., \& Whitmore, B. C. 2001, ApJ, 561, 727

Zhu, M., Seaquist, E. R., \& Kuno, N. 2003, ApJ, 588, 243 